\title{
Numerical solution and bifurcation analysis of nonlinear partial differential equations with extreme learning machines
}

\author{
Gianluca Fabiani ${ }^{1}$. Francesco Calabrò ${ }^{2} \cdot$ Lucia Russo $^{3} \cdot$ Constantinos Siettos $^{2}$ (D)
}

Received: 12 April 2021 / Revised: 14 July 2021 / Accepted: 19 August 2021 /

Published online: 11 October 2021

(C) The Author(s) 2021

\begin{abstract}
We address a new numerical method based on a class of machine learning methods, the socalled Extreme Learning Machines (ELM) with both sigmoidal and radial-basis functions, for the computation of steady-state solutions and the construction of (one-dimensional) bifurcation diagrams of nonlinear partial differential equations (PDEs). For our illustrations, we considered two benchmark problems, namely (a) the one-dimensional viscous Burgers with both homogeneous (Dirichlet) and non-homogeneous boundary conditions, and, (b) the oneand two-dimensional Liouville-Bratu-Gelfand PDEs with homogeneous Dirichlet boundary conditions. For the one-dimensional Burgers and Bratu PDEs, exact analytical solutions are available and used for comparison purposes against the numerical derived solutions. Furthermore, the numerical efficiency (in terms of numerical accuracy, size of the grid and execution times) of the proposed numerical machine-learning method is compared against central finite differences (FD) and Galerkin weighted-residuals finite-element (FEM) methods. We show that the proposed numerical machine learning method outperforms in terms of numerical accuracy both FD and FEM methods for medium to large sized grids, while provides equivalent results with the FEM for low to medium sized grids; both methods (ELM and FEM) outperform the FD scheme. Furthermore, the computational times required with the proposed
\end{abstract}

\footnotetext{
Constantinos Siettos

constantinos.siettos@unina.it

Gianluca Fabiani

gianluca.fabiani@unina.it

Francesco Calabrò

francesco.calabro@unina.it

Lucia Russo

lucia.russo@stems.cnr.it

1 Scuola Superiore Meridionale, Università degli Studi di Napoli Federico II, Naples, Italy

2 Dipartimento di Matematica e Applicazioni "Renato Caccioppoli”, Università degli Studi di Napoli "Federico II", Naples, Italy

3 IIstituto di Scienze e Tecnologie per l'Energia e la Mobilità Sostenibili, Consiglio Nazionale delle Ricerche, Naples, Italy
} 
machine learning scheme were comparable and in particular slightly smaller than the ones required with FEM.

Keywords Numerical analysis · Nonlinear partial differential equations · Numerical bifurcation analysis $\cdot$ Machine learning $\cdot$ Extreme learning machines

Mathematics Subject Classification $65 \mathrm{~N} 35 \cdot 65 \mathrm{~N} 75 \cdot 65 \mathrm{P} 30$

\section{Introduction}

The solution of partial differential equations (PDEs) with the aid of machine learning as an alternative to conventional numerical analysis methods can been traced back in the early ' 90 s. For example, Lagaris et al. [42] presented a method based on feedforward neural networks (FNN) that can be used for the numerical solution of linear and nonlinear PDEs. The method is based on the construction of appropriate trial functions, the analytical derivation of the gradient of the error with respect to the network parameters and collocation. The training of the FNN was achieved iteratively with the quasi-Newton BFGS method. Gonzalez-Garcia et al. [24] proposed a multilayer neural network scheme that resembles the Runge-Kutta integrator for the identification of dynamical systems described by nonlinear PDEs.

Nowadays, the exponentially increasing- over the last decades- computational power and recent theoretical advances, have allowed further developments at the intersection between machine learning and numerical analysis. In particular, on the side of the numerical solution of PDEs, the development of systematic and robust machine-learning methodologies targeting at the solution of large scale systems of nonlinear problems with steep gradients constitutes an open and challenging problem in the area. Very recently [51,52] addressed the use of numerical Gaussian Processes and Deep Neural Networks (DNNs) with collocation to solve time-dependent non-linear PDEs circumventing the need for spatial discretization of the differential operators. The proposed approach is demonstrated through the one-dimensional nonlinear Burgers, the Schrödinger and the Allen-Cahn equations. In [28], DNNs were used to solve high-dimensional nonlinear parabolic PDEs including the Black-Scholes, the HamiltonJacobi-Bellman and the Allen-Cahn equation. In [55], DNNs were used to approximate the solution of PDEs arising in engineering problems by exploiting the variational structure that may arise in some of these problems. In $[10,22,26]$ DNNs were used to solve highdimensional semi-linear PDEs; the efficiency of the method was compared against other deep learning schemes. In [62], the authors used FNN to solve modified high-dimensional diffusion equations: the training of the FNN is achieved iteratively using an unsupervised universal machine-learning solver. Most recently, in [21], the authors have used DNN to construct non-linear reduced-order models of time-dependent parametrized PDEs.

Over the last few years, extreme learning machines (ELMs) which belong to the more general family of random projection neural networks (RPNN) together with randomized and random vector functional link network (RVFLN) [35,47,57], echo-state neural networks and reservoir computing $[37,38,45,48,54]$ have been used as an alternative to other machine learning schemes, thus providing a good generalization at a low computational cost [34]. The idea behind ELMs is to randomly set the values of the weights between the input and hidden layer, the biases and the parameters of the activation/transfer functions and determine the weights between the last hidden and output layer by solving a least-squares problem. The solution of such a least-squares problem is the whole "training" procedure; hence, no iterative 
training is needed for ELMs, in contrast with what happens with the other aforementioned machine learning methods. Extensions to this basic scheme include multilayer ELMs [14,30, 59] and deep ELMs [60]. As with conventional neural networks, convolutional networks and deep learning, ELMs have been mainly used for classification purposes [4,11,12,32,59,61]. On the other hand, the use of ELMs for "traditional" numerical analysis tasks and in particular for the numerical solution of PDEs is still widely unexplored. Very recently in [20], it has been proposed a physics-informed ELM to solve stationary and time dependent linear PDEs, where the authors however report a failure of ELMs to deal, for example, with PDEs which solutions exhibit steep gradients. In [46], the authors used ELMs to solve ordinary and linear PDEs. In [18], the authors proposed a neural network for solving linear and nonlinear partial differential equations, based on the concepts of ELMs, domain decomposition and local neural networks: the domain is tessellated into sub-domains, and the solution on each subdomain is represented by a local shallow feed-forward neural network (ELMs). The authors solved the linear and the nonlinear 1D Helmotz equation, the 1D diffusion equation and the 1D viscous Burger's Equation. A comparison with Finite Elements with respect to the maximum error approximation as well as the computational time is also performed. Moreover, in [19], the authors addressed a modified batch intrinsic plasticity method for pre-training the random internal weights of the ELMs and applied the method to solve linear PDEs, namely the $2 \mathrm{D}$ Poisson equation and the $1 \mathrm{D}$ time-dependent problems of the wave equation and the diffusion equation. In [8], we have proposed an ELM scheme to deal with such steep gradients appearing in linear elliptic PDEs demonstrating through several benchmark problems that the proposed approach is efficient.

Here, we propose a problem-independent numerical scheme based on ELMs for the solution of steady-state problems of nonlinear 1D and 2D PDEs that exhibit sharp gradients. As nonlinear PDEs may also exhibit non-uniqueness and/or non-existence of solutions, we also show how one can use ELMs for the construction of (one-dimensional) bifurcation diagrams of PDEs. The efficiency of the proposed numerical scheme is demonstrated and discussed through two well-studied benchmark problems: the 1D viscous Burgers equation, a representative of the class of advection-diffusion problems and the 1D- and 2D Liouville-Bratu-Gelfand PDE, a representative of the class of reaction-diffusion problems. The numerical accuracy of the proposed scheme is compared against the analytical solutions and the exact locations of the limit points that are known for the one-dimensional PDEs, but also against the corresponding numerical approximations obtained with central Finite Differences (FD) and Galerkin Finite Elements Method (FEM).

\section{Extreme Learning Machines}

ELMs are a class of machine-learning techniques for defining functions derived by artificial neural networks (ANNs) with fixed internal weights and biases. Thus, ELMs have the same structure of a single hidden layer FNN with $N$ neurons. Next, we report the definition of ELM functions which we denote by $v(\boldsymbol{x}): \mathbb{R}^{d} \rightarrow \mathbb{R}$.

Definition 1 (ELM network with a single hidden layer) Assuming:

- An infinitely differentiable non polynomial function $\psi$, the activation (transfer) function for the neurons in the hidden layer.

- A randomly-generated matrix $A \in \mathbb{R}^{N \times d}$, made up of $N$ rows 


$$
A=\left(\begin{array}{c}
\alpha_{\mathbf{1}} \\
\boldsymbol{\alpha}_{\mathbf{2}} \\
\ldots \\
\boldsymbol{\alpha}_{N}
\end{array}\right), \quad \boldsymbol{\alpha}_{j}=\left(\alpha_{j, 1}, \alpha_{j, 2}, \ldots, \alpha_{j, d}\right) \in \mathbb{R}^{d},
$$

containing the internal weights connecting the input layer and the hidden layer.

- A randomly-generated vector $\beta \in \mathbb{R}^{N}$, containing the biases in the hidden layer.

Then, we say that $v$ is an ELM function with a single hidden layer, if there exists a choice of $w \in \mathbb{R}^{N}$, (the external weights vector between the hidden layer and the output layer) such that:

$$
v(\boldsymbol{x} ; A, \boldsymbol{\beta} ; \boldsymbol{w})=\sum_{j=1}^{N} w_{j} \psi\left(\boldsymbol{\alpha}_{j} \cdot \boldsymbol{x}+\beta_{j}\right),
$$

where $\boldsymbol{x} \in \mathbb{R}^{d}$ is the input column vector.

We remark that the regularity assumption in the above definition is not mandatory for the approximation properties, but in our case some regularity is needed to write the collocation method, thus for this case, we also briefly present the necessary theory. It is well-known, that for ANNs, where $A$ and $\boldsymbol{\beta}$ are not a-priori fixed, holds the universal approximation theorem, if $\psi$ is a non-polynomial function: the functional space is spanned by the basis functions $\left\{\psi\left(\boldsymbol{\alpha}_{j} \cdot \boldsymbol{x}+\beta_{j}\right), \boldsymbol{\alpha}_{j} \in \mathbb{R}^{d}, \beta_{j} \in \mathbb{R}\right\}$ that is dense in $L_{2}$. Moreover, with some regularity assumptions on the activation function(s), the approximation holds true also for the derivatives (see e.g. Theorem 3.1 and Theorem 4.1 in [49]). Besides, fixing $A$ and $\boldsymbol{\beta}$ a priori is not a limitation, because the universal approximation is still valid in the setting of ELMs (see Theorem 2 in [29]):

Theorem 1 (Universal approximation) Let the coefficients $\boldsymbol{\alpha}_{j}, \beta_{j}$ in the function sequence $\left\{\psi\left(\boldsymbol{\alpha}_{j} \cdot \boldsymbol{x}+\beta_{j}\right)\right\}_{j=1}^{N}$ be randomly generated according to any continuous sampling distribution and call $\tilde{v}^{N} \in \operatorname{span}\left\{\psi\left(\boldsymbol{\alpha}_{j} \cdot \boldsymbol{x}+\beta_{j}\right), j=1 \ldots N\right\}$ the ELM function determined by ordinary least square solution of $\left\|f(\boldsymbol{x})-\tilde{v}^{N}(\boldsymbol{x})\right\|$, where $f$ is a continuous function.

Then, one has with probability one that $\lim _{N \rightarrow \infty}\left\|f-\tilde{v}^{N}\right\|=0$.

We remark that in the ANN framework, the classical way is to optimize the parameters of the network (internal and external weights and biases) iteratively, e.g. by stochastic gradient descent algorithms that have a high computational cost and don't ensure a global but only local convergence. On the other hand, ELM networks are advantageous because the solution of an interpolation problem leads to a system of linear equations, where the only unknowns are the external weights $\boldsymbol{w}$. For example, consider $M$ points $\boldsymbol{x}_{i}$ such that $y_{i}=v\left(\boldsymbol{x}_{i}\right)$ for $i=1, \ldots, M$. In the ELM framework (1) the interpolation problem becomes:

$$
\sum_{j=1}^{N} w_{j} \psi_{j}\left(\boldsymbol{x}_{i}\right)=y_{i}, \quad i=1, \ldots, M,
$$

where $N$ is the number of neurons and $\psi_{j}(\boldsymbol{x})$ is used to denote $\psi\left(\boldsymbol{\alpha}_{j} \cdot \boldsymbol{x}+\beta_{j}\right)$. Thus, this is a system of $M$ equations and $N$ unknowns that in a matrix form can be we written as:

$$
S \boldsymbol{w}=\boldsymbol{y},
$$

where $\boldsymbol{y}=\left(y_{1}, \ldots, y_{M}\right) \in \mathbb{R}^{M}$ and $S \in \mathbb{R}^{M \times N}$ is the matrix with elements $(S)_{i j}=\psi_{j}\left(\boldsymbol{x}_{i}\right)$. If the problem is square $(N=M)$ and the parameters $\alpha_{j}$ and $\beta_{j}$ are chosen randomly, it 
can be proved that the matrix $S$ is invertible with probability 1 (see i.e. Theorem 1 [29]) and so, there is a unique solution, than can be numerically found; if one has to deal with an ill-conditioned matrix, one can still attempt to find a numerically robust solution by applying established numerical analysis methods suitable for such a case (e.g. by constructing the Moore-Penrose pseudoinverse using QR factorization or SVD). If the problem is underdetermined $(N>M)$, the linear system has (infinite) many solutions and can be solved by applying regularization in order to pick the solution, with e.g. the minimal $L_{2}$ norm. Such an approach provides the best solution to the optimization problem related to the magnitude of the calculated weights (see [33]).

Thus, in ELM networks, one has to choose the type of the activation/transfer function and the values of the internal weights and biases. Since the only limitation is that $\psi$ is a non-polynomial function, there are infinitely many choices. The most common choice are the sigmoidal functions (SF) (also referred as ridge functions or plane waves) and the radial basis functions (RBF) $[2,49]$.

Below, we describe the construction procedure and main features of the proposed ELM scheme, based on these two transfer functions. In the case of the logistic sigmoid transfer function, this investigation was made in our work for one-dimensional linear PDEs [8]. Here, we report the fundamental arguments and address a new numerical scheme for the numerical solution of one and two-dimensional steady-state nonlinear problems. Furthemore, we show how the proposed scheme can be coupled with tools from the numerical bifurcation theory for the construction of bifurcation diagrams.

\subsection{ELM with Sigmoidal Functions}

For the SF case, we select the logistic sigmoid, that is defined by

$$
\psi_{j}(\boldsymbol{x}) \equiv \sigma_{j}(\boldsymbol{x})=\frac{1}{1+\exp \left(-\boldsymbol{\alpha}_{j} \cdot \boldsymbol{x}-\beta_{j}\right)} .
$$

For this function, it is straightforward to compute the derivatives. In particular, the derivatives with respect to the $k$-th component of $\boldsymbol{x}, \boldsymbol{x}(k)$ are given by

$$
\begin{aligned}
\frac{\partial}{\partial \boldsymbol{x}(k)} \sigma_{j}(\boldsymbol{x}) & =\alpha_{j, k} \frac{\exp \left(z_{j}\right)}{\left(1+\exp \left(z_{j}\right)\right)^{2}} \\
\frac{\partial^{2}}{\partial x_{k}^{2}} \sigma_{j}(\boldsymbol{x}) & =\alpha_{j, k}^{2} \frac{\exp \left(z_{j}\right) \cdot\left(\exp \left(z_{j}\right)-1\right)}{\left(1+\exp \left(z_{j}\right)\right)^{3}},
\end{aligned}
$$

where $z_{j}=\boldsymbol{\alpha}_{j} \cdot \boldsymbol{x}+\beta_{j}$.

A crucial point in the ELM framework is how to fix the values of the internal weights and biases in a proper way. Indeed, despite the fact that theoretically any random choice should be good enough, in practice, it is convenient to define an appropriate range of values for the parameters $\alpha_{j, k}$ and $\beta_{j}$ that are strictly related to the selected activation function. For the one-dimensional case $(d=1)$, the logistic sigmoid $\sigma_{j}$ is a monotonic function such that:

$$
\begin{aligned}
& \alpha_{j, 1}>0 \Rightarrow \quad \lim _{x \rightarrow+\infty} \sigma_{j}(x)=1, \quad \lim _{x \rightarrow-\infty} \sigma_{j}(x)=0 \\
& \alpha_{j, 1}<0 \Rightarrow \quad \lim _{x \rightarrow+\infty} \sigma_{j}(x)=0, \quad \lim _{x \rightarrow-\infty} \sigma_{j}(x)=1 \text {. }
\end{aligned}
$$


This function has a inflection point, that we call center $c_{j}$ defined by the following property:

$$
\sigma_{j}\left(\alpha_{j, 1} c_{j}+\beta_{j}\right)=\frac{1}{2} .
$$

Now since $\sigma(0)=1 / 2$, the following relation between parameters holds:

$$
c_{j}=-\frac{\beta_{j}}{\alpha_{j, 1}} .
$$

Finally, $\sigma_{j}$ has a steep transition that is governed by the amplitude of $\alpha_{j, 1}$ : if $\left|\alpha_{j, 1}\right| \rightarrow+\infty$, then $\sigma_{j}$ approximates the Heaviside function, while if $\left|\alpha_{j, 1}\right| \rightarrow 0$, then $\sigma_{j}$ becomes a constant function. Now, since in the ELM framework, these parameters are fixed a priori, what one needs to avoid is to have some function that can be "useless"1 in the domain, say $I=[a, b]$. Therefore, for the one-dimensional case, our suggestion is to chose $\alpha_{j, 1}$ uniformly distributed as:

$$
\alpha_{j, 1} \sim \mathcal{U}\left(-\frac{N-55}{10|I|}, \frac{N+35}{10|I|}\right),
$$

where $N$ is the number of neurons in the the hidden layer and $|I|=b-a$ is the domain length. Moreover, we also suggest to avoid too small in module coefficients $a_{j}$ by setting:

$$
\left|\alpha_{j, 1}\right|>\frac{1}{2|I|} .
$$

Then, for the centers $c_{j}$, we select equispaced points in the domain $I$, that are given by imposing the $\beta_{j}$ s to be:

$$
\beta_{j}=-\alpha_{j, 1} \cdot c_{j}
$$

In the two-dimensional case $(d=2)$, we denote as $x=(x, y)^{T} \in \mathbb{R}^{2}$ the input and $A \in \mathbb{R}^{N \times 2}$ the matrix with rows $\boldsymbol{\alpha}_{j}=\left(\alpha_{j, 1}, \alpha_{j, 2}\right)$. Then, the condition (5) becomes:

$$
\sigma_{j}(x, y)=\sigma\left(\alpha_{j, 1} x+\alpha_{j, 2} y+\beta_{j}\right)=\frac{1}{2} .
$$

So, now we have:

$$
s \equiv y=-\frac{\alpha_{j, 1}}{\alpha_{j, 2}} x-\frac{\beta_{j}}{\alpha_{j, 2}}
$$

where $s$ is a straight line of inflection points that we call central direction. As the direction parallel to the central direction $\sigma_{j}$ is constant, while the orthogonal direction to $s$, the sigmoid $\sigma_{j}$ is exactly the one-dimensional logistic sigmoid. So considering one point $\boldsymbol{c}_{j}=\left(c_{j, 1}, c_{j, 2}\right)$ of the straight line $s$, we get the following relation between parameters:

$$
\beta_{j}=-\alpha_{j, 1} \cdot c_{j, 1}-\alpha_{j, 2} \cdot c_{j, 2} .
$$

Now, the difference with the one-dimensional case is the fact that in a domain $I^{2}=[a, b]^{2}$ discretized by a grid of $n \times n$ points, the number of neurons $N=n^{2}$ grows quadratically,

\footnotetext{
1 In Huang [31], it is suggested to take in $I=[-1,1]$ the $\alpha_{j, 1}$ randomly generated in the interval $[-1,1]$ and $\beta_{j}$ randomly generated in $[0,1]$. This construction leads to functions that are not well suited for our purposes: ad example if $\alpha_{j, 1}=0.1$ and $\beta_{j}=0.9$, the center is $c_{j}=-9$. Moreover if $\alpha_{j, 1}$ is small, the function $\phi_{j}$ is very similar to a constant function in $[-1,1]$, therefore this function is useless for our purposes.
} 
while the distance between two adjacent points decreases linearly, i.e. is given by $|I| /(n-1)$. Thus, for the two-dimensional case, we take $\alpha_{j, k}$ uniformly distributed as:

$$
\alpha_{j, k} \sim \mathcal{U}\left(-\frac{\sqrt{N}-60}{20|I|}, \frac{\sqrt{N}+40}{20|I|}\right), \quad k=1,2
$$

where $N$ is the number of neuron in the network and $|I|=b-a$.

\subsection{ELM with Radial Basis Functions}

Here, for the RBF case, we select the Gaussian kernel, that is defined as follows:

$$
\psi_{j}(\boldsymbol{x}) \equiv \varphi_{j}(\boldsymbol{x})=\exp \left(-\varepsilon_{j}^{2}\left\|\boldsymbol{x}-\boldsymbol{c}_{j}\right\|_{2}^{2}\right)=\exp \left(-\varepsilon_{j}^{2} \sum_{k=1}^{d}\left(\boldsymbol{x}(k)-c_{j, k}\right)^{2}\right),
$$

where $c_{j} \in \mathbb{R}^{d}$ is the center point and $\varepsilon_{j} \in \mathbb{R}$ is the inverse of the standard deviation. For such functions, we have:

$$
\begin{aligned}
\frac{\partial}{\partial \boldsymbol{x}(k)} \varphi_{j}(\boldsymbol{x}) & =-2 \varepsilon_{j}^{2}\left(x_{k}-c_{j, k}\right) \exp \left(-\varepsilon_{j}^{2} r_{j}^{2}\right), \\
\frac{\partial^{2}}{\partial x_{k}^{2}} \varphi_{j}(\boldsymbol{x}) & =-2 \varepsilon_{j}^{2}\left(1-2 \varepsilon_{j}^{2}\left(x_{k}-c_{j, k}\right)^{2}\right) \exp \left(-\varepsilon_{j}^{2} r_{j}^{2}\right),
\end{aligned}
$$

where $r_{j}=\left\|\boldsymbol{x}-\boldsymbol{c}_{j}\right\|_{2}$. In all the directions, the Gaussian kernel is a classical bell function such that:

$$
\lim _{\left\|\boldsymbol{x}-\boldsymbol{c}_{j}\right\| \rightarrow+\infty} \phi_{j}(\boldsymbol{x})=0, \quad \phi_{j}\left(\boldsymbol{c}_{j}\right)=1
$$

Moreover, the parameter $\varepsilon_{j}^{2}$ controls the steepness of the amplitude of the bell function: if $\varepsilon_{j} \rightarrow+\infty$, then $\phi_{j}$ approximates the Dirac function, while if $\varepsilon \rightarrow 0, \phi_{j}$ approximates a constant function. Thus, in the case of RBFs one can relate the role of $\varepsilon_{j}$ to the role of $\alpha_{j, k}$ for the case of SF. For RBFs, it is well known that the center has to be chosen as a point internal to the domain and also more preferable to be exactly a grid point, while the steepness parameter $\varepsilon$ is usually chosen to be the same for each function. Here, since we are embedding RBFs in the ELM framework, we take randomly the steepness parameter $\varepsilon_{j}$ in order to have more variability in the functional space, while for the centers $c_{j}$ we select equispaced points in the domain. Thus, as for the SF case, we set the parameters $\varepsilon_{j}^{2}$ random uniformly distributed as:

$$
\varepsilon_{j}^{2} \sim \mathcal{U}\left(\frac{1}{|I|}, \frac{N+65}{15|I|}\right),
$$

where $N$ denotes the number of neurons in the hidden layer and $|I|=b-a$ is the domain length. Besides, note that for the RBF case, it is trivial to extend the above into the multidimensional case, since $\varphi_{j}$ is already expressed with respect to the center. For the two-dimensional case, we do the same reasoning as for the SF taking:

$$
\varepsilon_{j}^{2} \sim \mathcal{U}\left(\frac{1}{2|I|}, \frac{\sqrt{N}+50}{30|I|}\right) .
$$




\section{Numerical Bifurcation Analysis of Nonlinear Partial Differential Equations with Extreme Learning Machines}

In this section, we introduce the general setting for the numerical solution and bifurcation analysis of nonlinear PDEs with ELMs based on basic numerical analysis concepts and tools (see e.g. $[7,9,13,23,50])$. Let's start from a nonlinear PDE of the general form:

$$
L u=f(u, \lambda) \text { in } \Omega,
$$

with boundary conditions:

$$
B_{l} u=g_{l}, \text { in } \partial \Omega_{l}, l=1,2, \ldots, m,
$$

where $L$ is the partial differential operator acting on $u, f(u, \lambda)$ is a nonlinear function of $u$ and $\lambda \in \mathbb{R}^{p}$ is the vector of model parameters, and $\left\{\partial \Omega_{l}\right\}_{l}$ denotes a partition of the boundary.

A numerical solution $\tilde{u}=\tilde{u}(\lambda)$ to the above problem at particular values of the parameters $\lambda$ is typically found iteratively by applying e.g. Newton-Raphson or matrix-free Krylovsubspace methods (Newton-GMRES) (see e.g. [39]) on a finite system of $M$ nonlinear algebraic equations. In general, these equations reflect some zero residual condition, or exactness equation, and thus the numerical solution that is sought is the optimal solution with respect to the condition in the finite dimensional space. Assuming that $\tilde{u}$ is fixed via the degrees of freedom $\boldsymbol{w} \in \mathbf{R}^{N}$ - we use the notation $\tilde{u}=\tilde{u}(\mathbf{w})$ - then these degrees of freedom are sought by solving:

$$
F_{k}\left(w_{1}, w_{2}, \ldots w_{j} \ldots w_{N} ; \lambda\right)=0, k=1,2, \ldots M .
$$

Many methods for the numerical solution of Eq. (8), (9) are written in the above form after the application of an approximation and discretization technique such as Finite Differences (FD), Finite Elements (FEM) and Spectral Expansion (SE), as we detail next.

The system of $M$ algebraic equations (10) is solved iteratively (e.g. by Newton's method), that is by solving until a convergence criterion is satisfied, the following linearized system:

$$
\nabla_{\boldsymbol{w}} \boldsymbol{F}\left(\boldsymbol{w}^{(n)}, \lambda\right) \cdot d \boldsymbol{w}^{(n)}=-\boldsymbol{F}\left(\boldsymbol{w}^{(n)}, \lambda\right), \quad \boldsymbol{w}^{(n+1)}=\boldsymbol{w}^{(n)}+d \boldsymbol{w}^{(n)} .
$$

$\nabla_{w} \boldsymbol{F}$ is the Jacobian matrix:

$$
\nabla_{\boldsymbol{w}} \boldsymbol{F}\left(\boldsymbol{w}^{(n)}, \lambda\right)=\left[\frac{\partial F_{k}}{\partial w_{j}}\right]_{\left.\right|_{\left(\boldsymbol{w}^{(n)}, \lambda\right)}}=\left[\begin{array}{cccccc}
\frac{\partial F_{1}}{\partial w_{1}} & \frac{\partial F_{1}}{\partial w_{2}} & \ldots & \frac{\partial F_{1}}{\partial w_{j}} & \ldots & \frac{\partial F_{1}}{\partial w_{N}} \\
\frac{\partial F_{2}}{\partial w_{1}} & \frac{\partial F_{2}}{\partial w_{2}} & \ldots & \frac{\partial F_{2}}{\partial w_{j}} & \ldots & \frac{\partial F_{2}}{\partial w_{N}} \\
\vdots & \vdots & \ddots & \vdots & \ddots & \vdots \\
\frac{\partial F_{k}}{\partial w_{1}} & \frac{\partial F_{k}}{\partial w_{2}} & \ldots & \frac{\partial F_{k}}{\partial w_{j}} & \ldots & \frac{\partial F_{k}}{\partial w_{N}} \\
\vdots & \vdots & \ddots & \vdots & \ddots & \vdots \\
\frac{\partial F_{M}}{\partial w_{1}} & \frac{\partial F_{M}}{\partial w_{2}} & \ldots & \frac{\partial F_{M}}{\partial w_{j}} & \ldots & \frac{\partial F_{M}}{\partial w_{N}}
\end{array}\right]_{\mid\left(\boldsymbol{w}^{(n)}, \lambda\right)}
$$

If the system is not square (i.e. when $M \neq N$ ), then at each iteration, one would perform e.g. QR-factorization of the Jacobian matrix

$$
\nabla_{\boldsymbol{w}} \boldsymbol{F}\left(\boldsymbol{w}^{(n)}, \lambda\right)=R^{T} Q^{T}=\left[\begin{array}{ll}
R_{1}^{T} & 0
\end{array}\right]\left[\begin{array}{c}
Q_{1}^{T} \\
Q_{2}^{T}
\end{array}\right],
$$

where $Q \in \mathbb{R}^{N \times N}$ is an orthogonal matrix and $R \in \mathbb{R}^{N \times M}$ is an upper triangular matrix. Then, the solution of Eq. (11) is given by:

$$
d \boldsymbol{w}^{(n)}=-Q_{1} R_{1}^{-1} \cdot \boldsymbol{F}\left(\boldsymbol{w}^{(n)}, \lambda\right)
$$


Branches of solutions in the parameter space past critical points on which the Jacobian matrix $\nabla F$ with elements $\frac{\partial F_{k}}{\partial w_{j}}$ becomes singular can be traced with the aid of numerical bifurcation analysis theory (see e.g. [15-17,25,40,41,56]). For example, solution branches past saddlenode bifurcations (limit/turning points) can be traced by applying the so called "pseudo" arc-length continuation method [9]. This involves the parametrization of both $\tilde{u}(\boldsymbol{w})$ and $\lambda$ by the arc-length $s$ on the solution branch. The solution is sought in terms of both $\tilde{u}(\boldsymbol{w} ; s)$ and $\lambda(s)$ in an iterative manner, by solving until convergence the following augmented system:

$$
\left[\begin{array}{cc}
\nabla_{\boldsymbol{w}} \boldsymbol{F} & \nabla_{\lambda} \boldsymbol{F} \\
\nabla_{\boldsymbol{w}} N & \nabla_{\lambda} N
\end{array}\right] \cdot\left[\begin{array}{c}
d \boldsymbol{w}^{(n)}(s) \\
d \lambda^{(n)}(s)
\end{array}\right]=-\left[\begin{array}{c}
\boldsymbol{F}\left(\boldsymbol{w}^{(n)}(s), \lambda(s)\right) \\
N\left(\tilde{u}\left(\boldsymbol{w}^{(n)} ; s\right), \lambda^{(n)}(s)\right)
\end{array}\right],
$$

where

$$
\nabla_{\lambda} \boldsymbol{F}=\left[\frac{\partial F_{1}}{\partial \lambda} \frac{\partial F_{2}}{\partial \lambda} \ldots \frac{F_{M}}{\partial \lambda}\right]^{T}
$$

and

$$
\begin{aligned}
N & \left(\tilde{u}\left(\boldsymbol{w}^{(n)} ; s\right), \lambda^{(n)}(s)\right) \\
= & \left(\tilde{u}\left(\boldsymbol{w}^{(n)} ; s\right)-\tilde{u}(\boldsymbol{w} ; s)_{-2}\right)^{T} \cdot \frac{\left(\tilde{u}(\boldsymbol{w})_{-2}-\tilde{u}(\boldsymbol{w})_{-1}\right)}{d s} \\
& +\left(\lambda^{(n)}(s)-\lambda_{-2}\right) \cdot \frac{\left(\lambda_{-2}-\lambda_{-1}\right)}{d s}-d s,
\end{aligned}
$$

is one of the choices for the so-called "pseudo arc-length condition" (for more details see e.g. $[9,16,23,25,41]) ; \tilde{u}(\boldsymbol{w})_{-2}$ and $\tilde{u}(\boldsymbol{w})_{-1}$ are two already found consequent solutions for $\lambda_{-2}$ and $\lambda_{-1}$, respectively and $d s$ is the arc-length step for which a new solution around the previous solution $\left(\tilde{u}(\boldsymbol{w})_{-2}, \lambda_{-2}\right)$ along the arc-length of the solution branch is being sought.

\subsection{Finite Differences and Finite Elements Cases: The Application of Newton's Method}

In FD methods, one aims to find the values of the solution per se (i.e. $u_{j}=w_{j}$ ) at a finite number of nodes within the domain. The operator in the differential problem (8) and the boundary conditions (9) are approximated by means of some finite difference operator: $L^{h} \approx L ; B_{l}^{h} \approx B_{l}$ : the finite operator reveals in some linear combination of the function evaluations for the differential part, while keeping non-linear requirement to be satisfied due to the presence of nonlinearities. Then, approximated equations are collocated in internal and boundary points $\boldsymbol{x}_{k}$ giving equations that can be written as residual equations (10).

With FEM and SE methods, the aim is to find the coefficients of a properly chosen basis function expansion of the solution within the domain such that the boundary conditions are satisfied precisely. In the Galerkin-FEM with Lagrangian basis (see e.g. [44,50]), the discrete counterpart seeks for a solution of Eq. (8), (9) in $N$ points $x_{j}$ of the domain $\Omega$ according to:

$$
u=\sum_{j=1}^{N} w_{j} \phi_{j}
$$

where the basis functions $\phi_{j}$ are defined so that they satisfy the completeness requirement and are such that $\phi_{j}\left(x_{k}\right)=\delta_{j k}$. This, again with the choice of nodal variables to be the function approximation at the points, gives that $u\left(x_{j}\right)=w_{j}$ are exactly the degrees of freedom for 
the method. Then, the numerical approximation of the solution is achieved by setting zero the weighted residuals $R_{k}, k=1,2, \ldots N$ defined as:

$$
R_{k}=\int_{\Omega}(L u-f(u, \lambda)) \phi_{k} d \Omega+\sum_{l=1}^{m} \int_{\partial \Omega_{k}}\left(B_{k} u-g_{l}\right) \phi_{l} d \sigma
$$

where the weighting functions $\phi_{i}$ are the same basis functions used in Eq. (15) for the approximation of $u$. The above constitutes a nonlinear system of $N$ algebraic equations that for a given set of values for $\lambda$ are solved by Newton-Raphson, thus solving until convergence the following linearized system seen in equation (11), where $R_{k}$ plays the role of $F_{k}$.

Note that the border rows and columns of the Jacobian matrix (12) are appropriately changed so that Eq. (11) satisfy the boundary conditions. Due to the construction of the basis functions, the Jacobian matrix is sparse, thus allowing the significant reduction of the computation cost for the solution of (11) at each Newton's iteration.

\subsection{Extreme Learning Machine Collocation: The Application of Newton's Method}

In an analogous manner to FE methods, Extreme Learning Machines aim at solving the problem (8), (9), using an approximation $\tilde{u}_{N}$ of $u$ with $N$ neurons as an ansatz. The difference is that, similarly to FD methods, the equations are constructed by collocating the solution on $M_{\Omega}$ points $\boldsymbol{x}_{i} \in \Omega$ and $M_{l}$ points $\boldsymbol{x}_{k} \in \partial \Omega_{l}$, where $\Omega_{l}$ are the parts of the boundary where boundary conditions are posed, see e.g. [3,50]:

$$
\begin{aligned}
L \tilde{u}_{N}\left(\boldsymbol{x}_{i} ; \boldsymbol{w}\right) & =f\left(\tilde{u}_{N}\left(\boldsymbol{x}_{i} ; \boldsymbol{w}\right), \lambda\right), \quad i=1, \ldots, M_{\Omega} \\
B_{l} \tilde{u}_{N}\left(\boldsymbol{x}_{k} ; \boldsymbol{w}\right) & =g_{l}\left(\boldsymbol{x}_{k}\right), \quad k=1, \ldots, M_{l}, \quad l=1, \ldots, m .
\end{aligned}
$$

Then, if we denote $M=M_{\Omega}+\sum_{l=1}^{m} M_{l}$, we have a system of $M$ nonlinear equations with $N$ unknowns that can be rewritten in a compact way as:

$$
F_{k}(\boldsymbol{w}, \lambda)=0, \quad k=1, \ldots, M,
$$

where for $k=1, \ldots, M_{\Omega}$, we have:

$$
F_{k}(\boldsymbol{w}, \lambda)=L\left(\sum_{i=1}^{N} w_{j} \psi\left(\boldsymbol{\alpha}_{j} \cdot \boldsymbol{x}_{i}+\beta_{j}\right)\right)-f\left(\sum_{i=1}^{N} w_{j} \psi\left(\boldsymbol{\alpha}_{j} \cdot \boldsymbol{x}_{i}+\beta_{j}\right)\right)=0,
$$

while for the $l$-th boundary condition, for $k=1, \ldots, M_{l}$ we have:

$$
F_{k}(\boldsymbol{w}, \lambda)=B_{l}\left(\sum_{i=1}^{N} w_{j} \psi\left(\boldsymbol{\alpha}_{j} \cdot \boldsymbol{x}_{i}+\beta_{j}\right)\right)-g\left(\sum_{i=1}^{N} w_{j} \psi\left(\boldsymbol{\alpha}_{j} \cdot \boldsymbol{x}_{i}+\beta_{j}\right)\right)=0 .
$$

At this system of non-linear algebraic equations, here we apply Newton's method (11). Notice that the application of the method requires the explicit knowledge of the derivatives of the functions $\psi$; in the ELM case as described, we have explicit formulae for these (see Eq. (4), (7)).

Remark 1 In our case, Newton's method is applied to non-squared systems. When the rank of the Jacobian is small, here we have chosen to solve the problem with the use of Moore-Penrose pseudo inverse of $\nabla_{\boldsymbol{w}} F$ computed by the SVD decomposition; as discussed above, another 
choice would be $Q R$-decomposition (13). This means that we cut off all the eigenvectors correlated to small eigenvalues, ${ }^{2}$ so:

$$
\nabla_{\boldsymbol{w}} \boldsymbol{F}=U \Sigma V^{T}, \quad\left(\nabla_{\boldsymbol{w}} \boldsymbol{F}\right)^{+}=V \Sigma^{+} U^{T}
$$

where $U \in \mathbb{R}^{M \times M}$ and $V \in \mathbb{R}^{N \times N}$ are the unitary matrices of left and right eigenvectors respectively, and $\Sigma \in \mathbb{R}^{M \times N}$ is the diagonal matrix of singular values. Finally, we can select $q \leq \operatorname{rank}(\nabla F)$ to get:

$$
\nabla_{\boldsymbol{w}} \boldsymbol{F}=U_{q} \Sigma_{q} V_{q}^{T}, \quad\left(\nabla_{\boldsymbol{w}} \boldsymbol{F}\right)^{+}=V_{q} \Sigma_{q}^{+} U_{q}^{T},
$$

where $U_{q} \in \mathbb{R}^{M \times q}$ and $V \in \mathbb{R}^{N \times q}$ and $\Sigma_{q} \in \mathbb{R}^{q \times q}$. Thus, the solution of Eq. (11) is given by:

$$
d \boldsymbol{w}^{(n)}=-V_{q} \Sigma_{q}^{+} U_{q}^{T} \cdot \boldsymbol{F}\left(\boldsymbol{w}^{(n)}, \lambda\right)
$$

Branches of solutions past turning points can be traced by solving the augmented, with the pseudo-arc-length condition, problem given by Eq. (14). In particular in (14), for the ELM framework (1), the term $\nabla_{\boldsymbol{w}} N$ becomes:

$$
\nabla_{\boldsymbol{w}} N=\boldsymbol{S}^{T} \frac{\left(\tilde{u}(\boldsymbol{w})_{-2}-\tilde{u}(\boldsymbol{w})_{-1}\right)}{d s}
$$

where $S$ is the collocation matrix defined in equation (2).

Remark 2 The three numerical methods (FD, FEM and ELM) are compared with respect to the dimension of the Jacobian matrix $J$, that in the case of FD and FEM is square and related to the number $N$ of nodes, i.e. $J \in \mathbb{R}^{N \times N}$, and in the case ELM is rectangular and related to both the number $M$ of collocation nodes and the number $N$ of neurons, i.e. $J \in \mathbb{R}^{M \times N}$. Actually, $N$ is the parameter related to the computational cost, i.e. the inversion of the $J$ for FD and FEM is done by LU factorization that has a computational cost of $O\left(N^{3}\right)$. For the ELM case, which leads to an under-determined system, the computational cost is related to the inversion of the matrix $J^{T} J \in \mathbb{R}^{N \times N}$, that therefore has the same leading computational cost $O\left(N^{3}\right)$. Moreover, if the solution is computed with the Moore-Penrose pseudo-inverse, the computational cost is based on Singular Value Decomposition which has a computational cost of $O\left(M N^{2}+M^{2} N\right)$. Finally, we make explicit that in all the rest of the paper, we use ELM networks with a number of $M$ of collocation points that is half the number $N$ of neurons in the hidden layer. Such a choice is justified by our previous work [8] that works better for linear PDEs with steep gradients. In general, we pinpoint that by increasing the number $M$ to be $\frac{2 N}{3}, \frac{3 N}{4}$, etc. ${ }^{3}$ one gets even better results.

Thus, in the case of collocation methods, such as FD and the proposed machine learning (ELMs) scheme, most of the computational cost is related to the solution of the linear system. In the case of isoparametric Galerkin methods, there is an extra computational cost related with the computation of the quantities of interest, such as the derivatives of the shape functions and the computation of the integrals.

2 The usual algorithm implemented in Matlab is that any singular value less than a tolerance is treated as zero: by default, this tolerance is set to $\max (\operatorname{size}(A)) * \operatorname{eps}(\operatorname{norm}(A))$.

3 The case $M=N$ can be solved only by the use of a (Moore-Penrose) pseudo-inverse (17), because the invertibility of the Jacobian of the nonlinear PDE operator cannot be guaranteed in advance. 


\section{Numerical Analysis Results: The Case Studies}

The efficiency of the proposed numerical scheme is demonstrated through two benchmark nonlinear PDEs, namely (a) the one dimensional nonlinear Burgers equation with Dirichlet boundary conditions and also mixed boundary conditions, and, (b) the one- and two-dimensional Liouville-Bratu-Gelfand problem. These problems have been widely studied as have been used to model and analyse the behaviour of many physical and chemical systems (see e.g. [1,6,9,23,27,36,53]).

All computations were made with a CPU Intel(R) Core(TM) i7-10750H CPU @ 2.60 GHz, RAM 16.0 GB using MATLAB R2020b.

In this section, we present some known properties of the proposed problems and provide details on their numerical solution with FD, FEM and the proposed machine learning (ELM) scheme with both logistic and Gaussian RBF transfer functions.

\subsection{The Nonlinear Viscous Burgers Equation}

Here, we consider the one-dimensional steady state viscous Burgers problem:

$$
v \frac{\partial^{2} u}{\partial x^{2}}-u \frac{\partial u}{\partial x}=0
$$

in the unit interval $[0,1]$, where $v>0$ denotes the viscosity. For our analysis, we considered two different sets of boundary conditions:

- Dirichlet boundary conditions

$$
u(0)=\gamma, u(1)=0, \gamma>0 ;
$$

- Mixed boundary conditions: Neumann condition on the left boundary and zero Dirichlet on the right boundary:

$$
\frac{\partial u}{\partial x}(0)=-\vartheta, u(1)=0, \vartheta>0 .
$$

The two sets of boundary conditions result to different behaviours (see [1,5]). We summarize in the next two lemmas some of the main results.

Lemma 1 (Dirichlet case) Consider Eq. (18) with boundary conditions given by (19). Moreover, take (notice that $\gamma \underset{v \rightarrow 0}{\longrightarrow} 1$ ):

$$
\gamma=\frac{2}{1+\exp \left(\frac{-1}{v}\right)}-1 .
$$

Then, the problem (18)-(19) has a unique solution given by:

$$
u(x)=\frac{2}{1+\exp \left(\frac{x-1}{v}\right)}-1 .
$$

We will use this test problem because the solution has a boundary layer and for this simple case, we can also implement and discuss the efficiency of a fixed point iteration by linearization, while in the mixed-boundaries case, we implement only the Newton's iterative procedure. 
Lemma 2 (Mixed case) Consider Eq. (18) with boundary conditions given by (20). The solution of the problem can be written as [1]:

$$
u(x)=\sqrt{2 c} \tanh \left(\frac{\sqrt{2 c}}{2 v}(1-x)\right),
$$

where $c$ is constant value which can be determined by the imposed Neumann condition. Then, for $\vartheta$ sufficiently small the viscous Burgers problem with mixed boundary conditions admits two solutions:

(a) a stable lower solution such that $\forall x \in(0,1)$ :

$$
u(x) \underset{\vartheta \rightarrow 0}{\longrightarrow} 0, \quad \frac{\partial u(x)}{\partial x} \underset{\vartheta \rightarrow 0}{\longrightarrow} 0,
$$

(b) an unstable upper solution $u(x)>0 \forall x \in(0,1)$ such that:

$$
\frac{\partial u(0)}{\partial x} \underset{\vartheta \rightarrow 0}{\longrightarrow} 0, \quad \frac{\partial u(1)}{\partial x} \underset{\vartheta \rightarrow 0}{\longrightarrow}-\infty,
$$

and

$$
\forall x \in(0,1), \quad u(x) \underset{\vartheta \rightarrow 0}{\longrightarrow} \infty
$$

Proof The spatial derivative of (22) is given by:

$$
\frac{\partial u(x)}{\partial x}=-\frac{c}{v} \operatorname{sech}^{2}\left(\frac{\sqrt{2 c}}{2 v}(1-x)\right) .
$$

(a) When $c \rightarrow 0$ then from Eq. (22), we get asymptotically the zero solution, i.e. $u(x) \rightarrow 0$, $\forall x \in(0,1)$ and from Eq. (23), we get $\frac{\partial u(x)}{\partial x} \rightarrow 0, \forall x \in(0,1)$. At $x=1$, the Dirichlet boundary condition $u(1)=0$ is satisfied exactly (see Eq. (22)), while at the left boundary $x=0$ the Neumann boundary condition is also satisfied as due to Eq. (23) and our assumption $(\vartheta \rightarrow 0): \frac{\partial u(0)}{\partial x}=-\vartheta \rightarrow 0$, when $c \rightarrow 0$.

(b) When $\frac{\partial u(1)}{\partial x} \rightarrow-\infty$, then (23) is satisfied $\forall x \in(0,1)$ when $c \rightarrow \infty$. In that case, at $x=0$, the Neumann boundary condition is satisfied as due to Eq. (23) is easy to prove that $\frac{\partial u(0)}{\partial x} \rightarrow 0$. Indeed, from Eq. (23):

$$
\lim _{c \rightarrow \infty} \frac{\partial u(x)}{\partial x}=-\lim _{c \rightarrow \infty} \frac{v}{\exp \frac{\sqrt{2 c}}{v}}=0 .
$$

Finally Eq. (22) gives $u(x) \rightarrow \infty, \forall x \in(0,1)$.

To better understand the behaviour of the unstable solution with respect to the left boundary condition, we can prove the following.

Corollary 1 Consider Eq. (18) with boundary conditions given by (20). For the non-zero solution, when $\vartheta=\epsilon \rightarrow 0$ the solution at $x=0$ goes to infinity with values:

$$
u(0)=v \log \left(\frac{v}{\epsilon}\right) \tanh \left(\frac{1}{2} \log \left(\frac{v}{\epsilon}\right)\right) .
$$


Proof By setting the value of $\vartheta$ in the Neumann boundary condition to be a very small number, i.e. $\vartheta=\epsilon \ll 1$, then from Eq. (24), we get that the slope of the analytical solution given by Eq. (23) is equal to $\epsilon$, when

$$
c=\frac{1}{2} v^{2} \log ^{2}\left(\frac{v}{\epsilon}\right) .
$$

Plugging the above into the analytical solution given by Eq. (22), we get Eq. (25).

The above findings imply also the existence of a limit point bifurcation with respect to $\vartheta$ that depends also on the viscosity. For example, as shown in [1], for $\vartheta>0$ and $v=1 / 10$, there are two equilibria arising due to a turning point at $\vartheta^{*}=0.087845767978$.

\subsubsection{Numerical Solution of the Burgers Equation with Finite Differences and Finite Elements}

The discretization of the one-dimensional viscous Burgers problem in $N$ points with secondorder central finite differences in the unit interval $0 \leq x \leq 1$ leads to the following system of $N-2$ algebraic equations $\forall x_{j}=(j-1) h, j=2, \ldots N-1, h=\frac{1}{N-1}$ :

$$
F_{j}(\boldsymbol{u})=\frac{v}{h^{2}}\left(u_{j+1}-2 u_{j}+u_{j-1}\right)-u_{j} \frac{u_{j+1}-u_{j-1}}{2 h}=0 .
$$

At the boundaries $x_{1}=0, x_{N}=1$, we have $u_{1}=\gamma, u_{N}=0$, respectively for the Dirichlet boundary conditions (19) and $u_{1}=\left(2 h \vartheta+4 u_{2}-u_{3}\right) / 3, u_{N}=0$, respectively for the mixed boundary conditions (20).

The above $N-2$ nonlinear algebraic equations are the residual equations (10) that are solved iteratively using Newton's method (11). The Jacobian (12) is now triagonal: at each $i$-th iteration, the non-null elements are given by:

$$
\frac{\partial F_{j}}{\partial u_{j-1}}=\frac{v}{h^{2}}+\frac{u_{j}}{2 h} ; \frac{\partial F_{j}}{\partial u_{j}}=-v \frac{2}{h^{2}}-\frac{u_{j+1}-u_{j-1}}{2 h} ; \frac{\partial F_{j}}{\partial u_{j+1}}=\frac{v}{h^{2}}-\frac{u_{j}}{2} .
$$

The Galerkin residuals (16) in the case of the one-dimensional Burgers equation read:

$$
R_{k}=\int_{0}^{1}\left(v \frac{\partial^{2} u(x)}{\partial x^{2}}-u \frac{\partial u(x)}{\partial x}\right) \phi_{k}(x) d x .
$$

By inserting the numerical solution (15) into Eq. (27) and by applying the Green's formula for integration, we get:

$$
\begin{aligned}
R_{k}= & \left.v \phi_{k}(x) \frac{d u}{d x}\right|_{0} ^{1}-v \sum_{j=1}^{N} u_{j} \int_{0}^{1} \frac{d \phi_{j}(x)}{d x} \frac{d \phi_{k}(x)}{d x} d x \\
& -\int_{0}^{1} \sum_{j=1}^{N} u_{j} \phi_{j}(x) \sum_{j=1}^{N} u_{j} \frac{d \phi_{j}(x)}{d x} \phi_{k}(x) d x .
\end{aligned}
$$


At the above residuals, we have to impose the boundary conditions. If Dirichlet boundary conditions (19) are imposed, Eq. (28) becomes:

$$
\begin{aligned}
R_{k}= & -v \sum_{j=1}^{N} u_{j} \int_{0}^{1} \frac{d \phi_{j}(x)}{d x} \frac{d \phi_{k}(x)}{d x} d x \\
& -\int_{0}^{1} \sum_{j=1}^{N} u_{j} \phi_{j}(x) \sum_{j=1}^{N} u_{j} \frac{d \phi_{j}(x)}{d x} \phi_{k}(x) d x .
\end{aligned}
$$

In the case of the mixed boundary conditions (20), Eq. (28) becomes:

$$
\begin{aligned}
R_{k}= & v \vartheta \phi_{k}(0)-v \sum_{j=1}^{N} u_{j} \int_{0}^{1} \frac{d \phi_{j}(x)}{d x} \frac{d \phi_{k}(x)}{d x} d x \\
& -\int_{0}^{1} \sum_{j=1}^{N} u_{j} \phi_{j}(x) \sum_{j=1}^{N} u_{j} \frac{d \phi_{j}(x)}{d x} \phi_{k}(x) d x .
\end{aligned}
$$

In this paper, we use a $P^{2}$ Finite Element space, thus quadratic basis functions using an affine element mapping in the interval $[0,1]^{d}$. For the computation of the integrals, we used the Gauss quadrature numerical scheme: for the one-dimensional case, we used the three-points gaussian rule:

$$
\left\{\left(\frac{1}{2}-\sqrt{\frac{3}{20}}, \frac{5}{18}\right),\left(0.5, \frac{8}{18}\right),\left(\frac{1}{2}+\sqrt{\frac{3}{20}}, \frac{5}{18}\right)\right\}
$$

When writing Newton's method (11), the elements of the Jacobian matrix for both (29) and (30) are given by:

$$
\frac{\partial R_{k}}{\partial u_{j}}=-v \int_{0}^{1} \frac{d \phi_{j}(x)}{d x} \frac{d \phi_{k}(x)}{d x} d x-2 \int_{0}^{1} \sum_{j=1}^{N} u_{j} \phi_{j}(x) \frac{d \phi_{j}(x)}{d x} \phi_{k}(x) d x .
$$

Finally, with all the above, the Newton's method (11) involves the iterative solution of a linear system. For the Dirichlet problem this becomes:

$$
\left[\begin{array}{cccccc}
1 & 0 & \ldots & 0 & \ldots & 0 \\
\frac{\partial R_{2}}{\partial u_{1}} & \frac{\partial R_{2}}{\partial u_{2}} & \ldots & \frac{\partial R_{2}}{\partial u_{j}} & \ldots & \frac{\partial R_{2}}{\partial u_{N}} \\
\vdots & \vdots & \ddots & \vdots & \ddots & \vdots \\
\frac{\partial R_{k}}{\partial u_{1}} & \frac{\partial R_{k}}{\partial u_{2}} & \ldots & \frac{\partial R_{k}}{\partial u_{j}} & \ldots & \frac{\partial R_{k}}{\partial u_{N}} \\
\vdots & \vdots & \ddots & \vdots & \ddots & \vdots \\
0 & 0 & \ldots & 0 & \ldots & 1
\end{array}\right]_{\mid u^{(n)}} \quad \cdot\left[\begin{array}{c}
d u_{1}^{(n)} \\
d u_{2}^{(n)} \\
\vdots \\
d u_{j}^{(n)} \\
\vdots \\
d u_{N}^{(n)}
\end{array}\right]=-\left[\begin{array}{c}
0 \\
R_{2} \\
\vdots \\
R_{k} \\
\vdots \\
0
\end{array}\right]_{\mid u^{(n)}}
$$


while for the problem with the mixed boundary conditions, at each iteration, we need to solve the following system:

$$
\left[\begin{array}{cccccc}
\frac{\partial R_{1}}{\partial u_{1}} & \frac{\partial R_{1}}{\partial u_{2}} & \ldots & \frac{\partial R_{1}}{\partial u j} & \cdots & \frac{\partial R_{1}}{\partial u_{N}} \\
\frac{\partial R_{2}}{\partial u_{1}} & \frac{\partial R_{2}}{\partial u_{2}} & \ldots & \frac{\partial R_{2}}{\partial u_{j}} & \cdots & \frac{\partial R_{2}}{\partial u_{N}} \\
\vdots & \vdots & \ddots & \vdots & \ddots & \vdots \\
\frac{\partial R_{k}}{\partial u_{1}} & \frac{\partial R_{k}}{\partial u_{2}} & \ldots & \frac{\partial R_{k}}{\partial u_{j}} & \ldots & \frac{\partial R_{k}}{\partial u_{N}} \\
\vdots & \vdots & \ddots & \vdots & \ddots & \vdots \\
0 & 0 & \ldots & 0 & \ldots & 1
\end{array}\right]_{u^{(n)}}\left[\begin{array}{c}
d u_{1}^{(n)} \\
d u_{2}^{(n)} \\
\vdots \\
d u_{j}^{(n)} \\
\vdots \\
d u_{N}^{(n)}
\end{array}\right]=-\left[\begin{array}{c}
R_{1} \\
R_{2} \\
\vdots \\
R_{k} \\
\vdots \\
0
\end{array}\right]_{\mid u^{(n)}}
$$

\subsubsection{Numerical Solution of the Burgers Equation with Extreme Learning Machine Collocation}

Collocating the ELM network function for the one-dimensional Burgers equation leads to the following nonlinear algebraic system for $i=2, \ldots, M-1$ :

$$
F_{i}(\boldsymbol{w}, v)=v \sum_{j=1}^{N} w_{j} \frac{d^{2} \psi_{j}\left(x_{i}\right)}{d x^{2}}-\left(\sum_{j=1}^{N} w_{j} \psi_{j}\left(x_{i}\right)\right)\left(\sum_{j=1}^{N} w_{j} \frac{d \psi_{j}\left(x_{i}\right)}{d x}\right)=0 .
$$

Then, the imposition of the boundary conditions (19) gives:

$$
F_{1}(\boldsymbol{w}, v)=\sum_{j=1}^{N} w_{j} \psi_{j}(0)-\gamma=0, \quad F_{M}(\boldsymbol{w}, v)=\sum_{j=1}^{N} w_{j} \psi_{j}(1)=0,
$$

while boundary conditions (20) lead to:

$$
F_{1}(\boldsymbol{w}, v)=\sum_{j=1}^{N} w_{j} \frac{d \psi_{j}(0)}{d x}+\vartheta=0, \quad F_{M}(\boldsymbol{w}, v)=\sum_{j=1}^{N} w_{j} \psi_{j}(1)=0 .
$$

These equations are the residual equations (10) that we solve by Newton's method (11). The elements of the Jacobian matrix $\nabla_{\boldsymbol{w}} \boldsymbol{F}$ are given by:

$$
\frac{\partial F_{i}}{\partial w_{j}}=v \frac{d^{2} \psi_{j}\left(x_{i}\right)}{d x^{2}}-\psi_{j}\left(x_{i}\right)\left(\sum_{j=1}^{N} w_{j} \frac{d \psi_{j}\left(x_{i}\right)}{d x}\right)-\left(\sum_{j=1}^{N} w_{j} \psi_{j}\left(x_{i}\right)\right) \frac{d \psi_{j}\left(x_{i}\right)}{d x} .
$$

For $i=2, \ldots, M-1$ and due to the Dirichlet boundary conditions (35), we have:

$$
\frac{\partial F_{1}}{\partial w_{j}}(\boldsymbol{w}, \lambda)=\psi_{j}(0) \quad \frac{\partial F_{M}}{\partial w_{j}}(\boldsymbol{w}, \lambda)=\psi_{j}(1) .
$$

On the other hand, due to the mixed boundary conditions given by (36), we get:

$$
\frac{\partial F_{1}}{\partial w_{j}}(\boldsymbol{w}, \lambda)=\frac{d \psi_{j}(0)}{d x} \quad \frac{\partial F_{M}}{\partial w_{j}}(\boldsymbol{w}, \lambda)=\psi_{j}(1)
$$

At this point, the application of Newton's method (11) using the exact computation of the derivatives of the basis functions is straightforward (see (4) and (7)). 


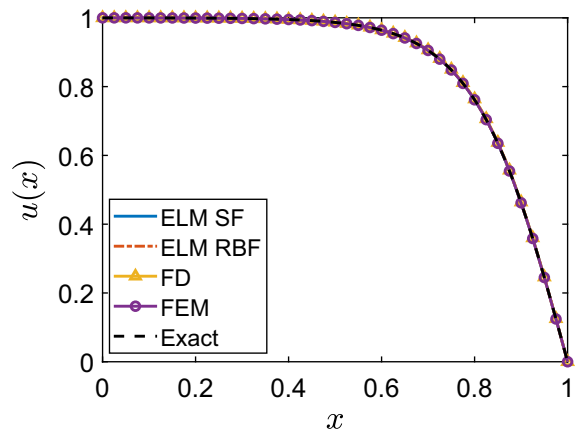

(a)

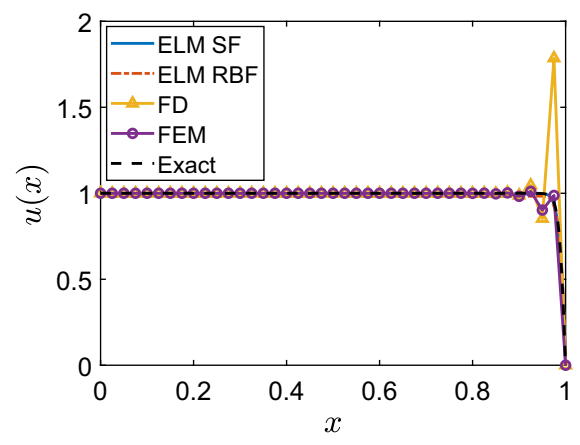

(c)

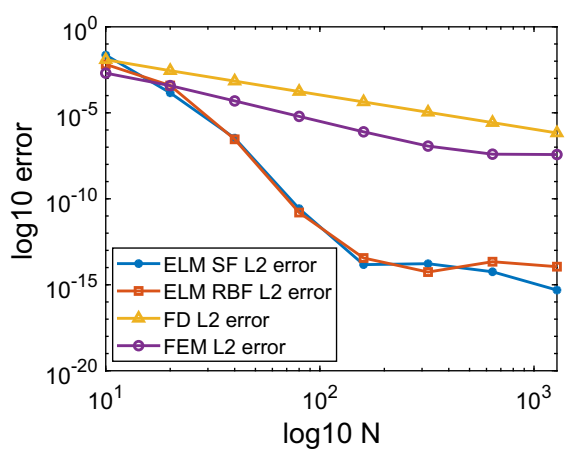

(b)

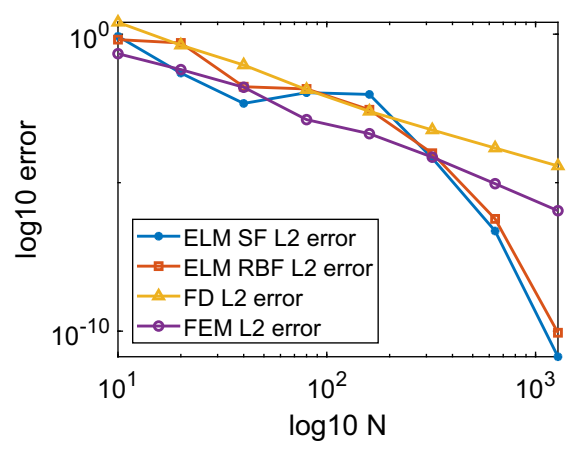

(d)

Fig. 1 Numerical solution and accuracy of the FD, FEM and the proposed machine learning (ELM) schemes for the one-dimensional viscous Burgers problem with Dirichlet boundary conditions (18), (19), (a,b) with viscosity $v=0.1$ : (a) Solutions for a fixed problem size $N=40$; (b) $L_{2}$-norm of differences with respect to the exact solution (21) for various problem sizes. (c,d) with viscosity $v=0.007$ : (c) Solutions for a fixed problem size $N=40$; (d) $L_{2}$-norm errors with respect to the exact solution for various problem sizes

\subsubsection{Numerical Results}

In all the computations with FD, FEM and the proposed machine learning (ELM) scheme, the convergence criterion for Newton's iterations was the $L_{2}$ norm $^{4}$ of the relative error between the solutions resulting from successive iterations; the convergence tolerance was set to $10^{-6}$. In fact, for all methods, Newton's method converged quadratically also up to the order of $10^{-10}$, when the bifurcation parameter was not close to zero where the solution of both Burgers with mixed boundary conditions and Bratu problems goes asymptotically to infinity. The exact solutions that are available for the one-dimensional Burgers and Bratu problems are derived using Newton's method with a convergence tolerance of $10^{-12}$.

First, we present the numerical results for the Burgers equation (18) with Dirichlet boundary conditions (19). Recall that for this case, the exact solution is available (see Eq. (21)). For our illustrations, we have selected two different values for the viscosity, namely $v=0.1$ and $v=0.007$. Results were obtained with Newton's iterations starting from an initial guess that

4 The relative error is the $L_{2}$-norm of the difference between two successive solutions $\left\|u(\boldsymbol{w})_{-2}-u(\boldsymbol{w})_{-1}\right\|_{2}$. In particular for the ELM framework is given by $\left\|S^{T} \cdot\left(\boldsymbol{w}_{-2}-\boldsymbol{w}_{-1}\right)\right\|_{2}$, where $S$ is the collocation matrix defined in Eq. (2). 
Table 1 Execution times (s) for the Burgers equation (18) with Dirichlet boundary conditions (19) and $v=0.1$

\begin{tabular}{|c|c|c|c|c|c|c|}
\hline \multirow[b]{2}{*}{$\mathrm{N}$} & \multicolumn{3}{|l|}{ ELM SF } & \multicolumn{3}{|l|}{ ELM RBF } \\
\hline & $5 \%$ & Mean & $95 \%$ & $5 \%$ & mean & $95 \%$ \\
\hline 80 & $2.73 e-03$ & $4.70 \mathrm{e}-03$ & $4.38 \mathrm{e}-03$ & $2.31 \mathrm{e}-03$ & $2.67 \mathrm{e}-03$ & $3.16 \mathrm{e}-03$ \\
\hline 160 & $8.46 \mathrm{e}-03$ & $9.97 \mathrm{e}-03$ & $1.12 \mathrm{e}-02$ & $7.16 \mathrm{e}-03$ & $8.20 \mathrm{e}-03$ & $9.08 \mathrm{e}-03$ \\
\hline 320 & $3.72 \mathrm{e}-02$ & $4.23 \mathrm{e}-02$ & $4.60 \mathrm{e}-02$ & $3.52 \mathrm{e}-02$ & $3.89 \mathrm{e}-02$ & $4.28 \mathrm{e}-02$ \\
\hline \multirow[t]{2}{*}{640} & $1.60 \mathrm{e}-01$ & $1.67 \mathrm{e}-01$ & $1.75 \mathrm{e}-01$ & $1.56 \mathrm{e}-01$ & $1.69 \mathrm{e}-01$ & $1.97 \mathrm{e}-01$ \\
\hline & \multicolumn{3}{|l|}{$\underline{\text { FD }}$} & \multicolumn{3}{|l|}{ FEM } \\
\hline $\mathrm{N}$ & $5 \%$ & mean & $95 \%$ & $5 \%$ & mean & $95 \%$ \\
\hline 80 & $1.72 \mathrm{e}-04$ & $3.37 \mathrm{e}-04$ & $3.48 \mathrm{e}-04$ & $2.33 \mathrm{e}-02$ & $2.49 \mathrm{e}-02$ & $2.76 \mathrm{e}-02$ \\
\hline 160 & $4.31 \mathrm{e}-04$ & $4.55 \mathrm{e}-04$ & $5.26 \mathrm{e}-04$ & $5.68 \mathrm{e}-02$ & $6.39 \mathrm{e}-02$ & $6.95 e-02$ \\
\hline 320 & $1.29 \mathrm{e}-03$ & $1.33 \mathrm{e}-03$ & $1.44 \mathrm{e}-03$ & $1.22 \mathrm{e}-01$ & $1.24 \mathrm{e}-01$ & $1.31 \mathrm{e}-01$ \\
\hline 640 & $1.05 \mathrm{e}-02$ & $1.10 \mathrm{e}-02$ & $1.16 \mathrm{e}-02$ & $3.34 \mathrm{e}-01$ & $3.40 \mathrm{e}-01$ & $3.55 \mathrm{e}-01$ \\
\hline
\end{tabular}

is a linear segment that satisfies the boundary conditions. Figure 1 shows the corresponding computed solutions for a fixed size $N=40$ as well as the relative errors with respect to the exact solution. As it is shown, the proposed machine learning scheme outperforms both the FD and FEM schemes for medium to large sizes of the grid; from low to medium sizes of the grid, all methods perform equivalently. However, as shown in Fig. 1c, for $v=0.007$, and the particular choice of the size $(N=40)$, the FD scheme fails to approximate sufficiently the steep-gradient appearing at the right boundary. Table 1, summarizes the execution times of the four methods (ELM SF, ELM RBF, FD and FEM), when applied for the solution of the Burgers equation (18) with Dirichlet conditions (19) and $v=0.1$ for various sizes of the grid. The computations are performed 100 times and we also provide the 5\% and $95 \%$ percentiles. For all practical means, the execution times obtained with ELMS are comparable with the ones obtained with FD and FEM (with the ones obtained with the proposed machine learning scheme to be slightly faster that the ones obtained with FEM), while, as seen, numerical approximation accuracy is better in the ELMs case; the execution times when using the FD are smaller but as shown the FD scheme fails to approximate solutions with steep gradients while its numerical accuracy is generally lower when compared with FEM and the proposed machine learning scheme.

Then, we considered the case of the non-homogeneous Neumann condition on the left boundary (18)- (20); here, we have set $v=1 / 10$. In this case, the solution is not unique and the resulting bifurcation diagram obtained with FD, FEM and the proposed machine learning (ELM) scheme is depicted in Fig. 2. In Table 2, we report the error between the value of the bifurcation point as computed with FD, FEM and the proposed machine learning (ELM) scheme for various problem sizes $N$, with respect to the exact value of the bifurcation point (occurring for the particular choice of viscosity at $\vartheta^{*}=0.087845767978$ ). The location of the bifurcation point for all numerical methods was estimated by fitting a parabola around the four points (two on the lower and two on the upper branch) of the largest values of $\lambda$ as obtained by the pseudo-arc-length continuation. As shown, the proposed ELM scheme performs equivalently to FEM for low to medium sizes of the grid, thus outperforming FEM for medium to large grid sizes; both methods FEM and the proposed machine learning (ELM) scheme outperform FD for all sizes of the grid. 


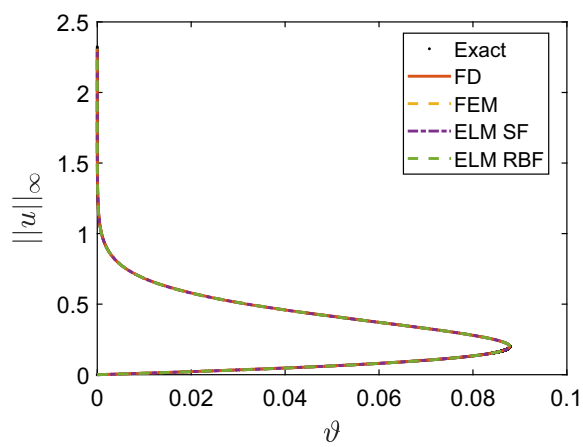

(a)

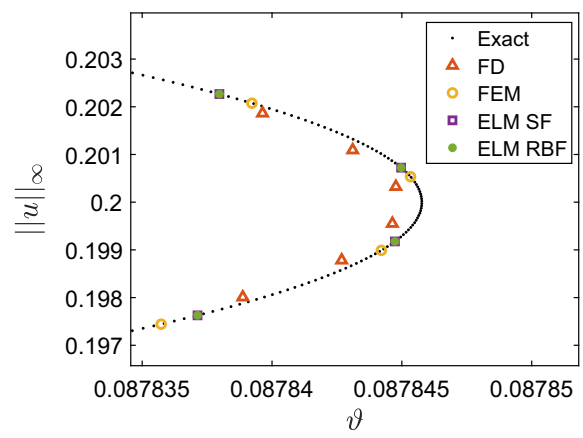

(b)

Fig. 2 a One-dimensional Burgers equation (18) with mixed boundary conditions (20). Bifurcation diagram with respect to the Neumann boundary value $\theta$ as obtained for $v=1 / 10$, with FD, FEM and ELM schemes with a fixed problem size $N=400$; $\mathbf{b}$ Zoom near the turning point

Table 2 One-dimensional Burgers equation (18) with mixed boundary conditions (20)

\begin{tabular}{lllll}
\hline $\mathrm{N}$ & FD & FEM & ELM SF & ELM RBF \\
\hline 20 & $-3.32 \mathrm{e}-04$ & $-4.86 \mathrm{e}-09$ & $2.75 \mathrm{e}-08$ & $-4.37 \mathrm{e}-06$ \\
50 & $-5.35 \mathrm{e}-05$ & $-7.67 \mathrm{e}-09$ & $-2.06 \mathrm{e}-09$ & $-2.14 \mathrm{e}-09$ \\
100 & $-1.34 \mathrm{e}-05$ & $-2.16 \mathrm{e}-09$ & $-9.84 \mathrm{e}-09$ & $-9.85 \mathrm{e}-09$ \\
200 & $-3.34 \mathrm{e}-06$ & $-5.93 \mathrm{e}-09$ & $-9.62 \mathrm{e}-09$ & $-9.61 \mathrm{e}-09$ \\
400 & $-8.35 \mathrm{e}-07$ & $4.15 \mathrm{e}-09$ & $9.38 \mathrm{e}-10$ & $9.33 \mathrm{e}-10$ \\
\hline
\end{tabular}

Comparative results with respect to the error between the estimated value of the turning point as obtained with FD, FEM and proposed machine learning (ELM) schemes and the exact value of the turning point at $\vartheta^{*}=0.087845767978$ for $v=1 / 10$. The value of the turning point was estimated by fitting a parabola around the four points with the largest $\lambda$ values as obtained by the arc-length continuation

In this case, steep gradients arise at the right boundary related to the presence of the upper unstable solution, as discussed in Lemma 2 and Corollary 1. In Table 3, we report the error between the numerically computed and the exact analytically obtained value (see Eq. (22)) at $x=0$ when the value of boundary condition $\vartheta$ at the left boundary is $\vartheta=10^{-6}$. Again as shown, near the left boundary, the proposed ELM scheme outperforms both FEM and FD for medium to larger sizes of the grid.

Remark 3 (Linearization of the Burgers equation for its numerical solution.) For the numerical solution of the Burgers equation (18) with boundary conditions given by (19), one can also consider the following simple iterative procedure that linearizes the equation: 
Table 3 One-dimensional Burgers equation (18) with mixed boundary conditions (20)

\begin{tabular}{lllll}
\hline $\mathrm{N}$ & FD & FEM & ELM SF & ELM RBF \\
\hline 20 & $-1.81 \mathrm{e}-01$ & $2.05 \mathrm{e}-02$ & $-6.55 \mathrm{e}-01$ & $-6.14 \mathrm{e}-01$ \\
50 & $-2.66 \mathrm{e}-02$ & $7.67 \mathrm{e}-04$ & $-5.83 \mathrm{e}-01$ & $-6.09 \mathrm{e}-01$ \\
100 & $-6.52 \mathrm{e}-03$ & $1.58 \mathrm{e}-04$ & $-2.00 \mathrm{e}-01$ & $-1.05 \mathrm{e}-01$ \\
200 & $-1.61 \mathrm{e}-03$ & $8.99 \mathrm{e}-05$ & $-2.50 \mathrm{e}-06$ & $-5.05 \mathrm{e}-06$ \\
400 & $-4.00 \mathrm{e}-04$ & $6.28 \mathrm{e}-05$ & $-3.47 \mathrm{e}-06$ & $-9.52 \mathrm{e}-06$
\end{tabular}

Comparative results with respect to the error between the computed solution (at $x=0$ ) with FD, FEM and proposed machine learning (ELMs) scheme (with both sigmoidal and radial basis functions) and the exact solution $u(0)=1.798516682636303$ (see Eq. (22)) for $\vartheta=1 e-6$ (the value of the Neumann condition at the left boundary)

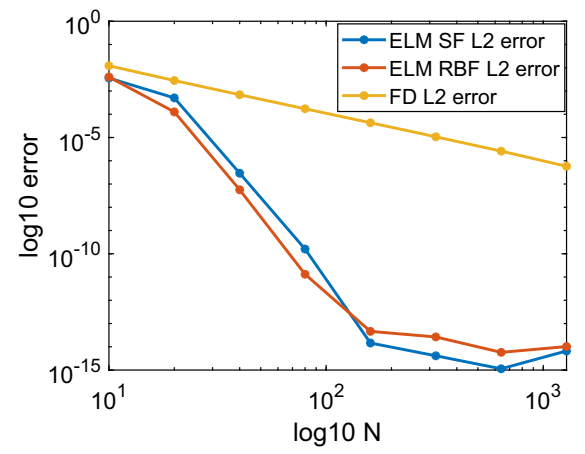

(a)

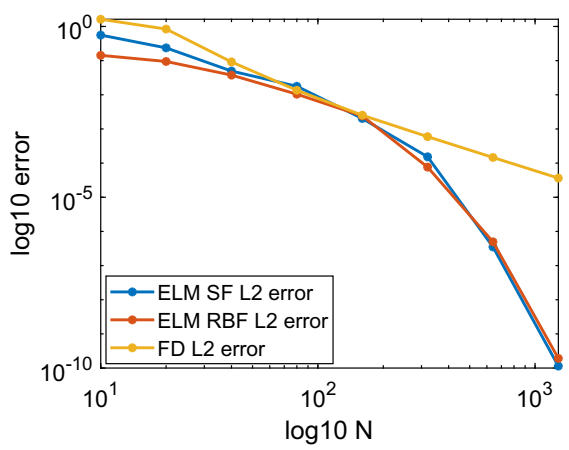

(b)

Fig. 3 Numerical accuracy of FD and ELM schemes with respect to the exact solution, for the case of the one-dimensional Burgers equation (18) with Dirichlet boundary conditions given by (19), as obtained by the fixed point scheme described in Remark 3 for $\mathbf{a} v=0.1$ and $\mathbf{b} v=0.007$. We depict the $L_{2}$-norm of the difference between the solutions obtained with FD and the proposed machine learning (ELMs) scheme and the exact solution (21)

$$
\left\{\begin{array}{l}
\text { Given } u^{(0)}, \text { do until convergence } \\
\text { find } u^{(k)} \text { such that } v \frac{\partial^{2} u^{(k)}}{\partial x^{2}}-u^{(k-1)} \frac{\partial u^{(k)}}{\partial x}=0 .
\end{array}\right.
$$

In this way, the nonlinear term becomes a linear advection term with a non-constant coefficient given by the evaluation of $u$ at the previous iteration. This results to a fixed point scheme. Such linearized equations can be easily solved, being linear elliptic equations, and thus in this case one can perform the analysis for linear systems presented in [8]. The results of this procedure are depicted in Fig. 3.

We point out that such iterations converge generally very slowly and, what is most important from our point of view, is that convergence is obtained only for a very "good" guess of the solution. 


\subsection{The One- and Two-Dimensional Liouville-Bratu-Gelfand Problem}

The Liouville-Bratu-Gelfand model arises in many physical and chemical systems. It is an elliptic partial differential equation which in its general form is given by [6]:

$$
\Delta u(\boldsymbol{x})+\lambda e^{u(x)}=0 \quad x \in \Omega,
$$

with homogeneous Dirichlet conditions

$$
u(\boldsymbol{x})=0, \boldsymbol{x} \in \partial \Omega \text {. }
$$

The domain that we consider here is the $\Omega=[0,1]^{d}$ in $R^{d}, d=1,2$.

The one-dimensional problem admits an analytical solution given by [43]:

$$
u(x)=2 \ln \frac{\cosh \theta}{\cosh \theta(1-2 x)}, \text { where } \theta \text { is such that } \cosh \theta=\frac{4 \theta}{\sqrt{2 \lambda}} .
$$

It can be shown that when $0<\lambda<\lambda_{c}$, the problem admits two branches of solutions that meet at $\lambda_{c} \sim 3.513830719$, a limit point (saddle-node bifurcation) that marks the onset of two branches of solutions with different stability, while beyond that point no solutions exist.

For the two-dimensional problem, to the best of our knowledge, no such (as in the onedimensional case) exact analytical solution exist that is verified by the numerical results that have been reported in the literature (e.g. $[9,27]$ ), in which the authors report the value of the turning at $\lambda_{c} \sim 6.808124$.

\subsubsection{Numerical Solution with Finite Differences and Finite Elements}

The discretization of the one-dimensional problem in $N$ points with central finite differences at the unit interval $0 \leq x \leq 1$ leads to the following system of $N-2$ algebraic equations $\forall x_{j}=(j-1) h, j=2, \ldots N-1, h=\frac{1}{N-1}$ :

$$
F_{j}(u)=\frac{1}{h^{2}}\left(u_{j+1}-2 u_{j}+u_{j-1}\right)+\lambda e^{u_{j}}=0,
$$

where, at the boundaries $x_{1}=0, x_{N}=1$, we have $u_{1}=u_{N}=0$.

The solution of the above $N-2$ nonlinear algebraic equations is obtained iteratively using the Newton-Raphson method. The Jacobian is now tridiagonal; at each $n$-th iteration, the elements at the main diagonal are given by $\frac{\partial F_{j}}{\partial u_{j}}{ }^{(n)}=-\frac{2}{h^{2}}+\lambda e^{u_{j}^{(n)}}$ and the elements of the first diagonal above and the first diagonal below are given by ${\frac{\partial F_{j+1}}{\partial u_{j}}}^{(n)}={\frac{\partial F_{j}}{\partial u_{j+1}}}^{(n)}=\frac{1}{h^{2}}$, respectively.

The discretization of the two-dimensional Bratu problem in $N \times N$ points with central finite differences on the square grid $0 \leq x, y \leq 1$ with zero boundary conditions leads to the following system of $(N-2) \times(N-2)$ algebraic equations $\forall\left(x_{i}=(i-1) h, y_{j}=\right.$ $(j-1) h), i, j=2, \ldots N-1, h=\frac{1}{N-1}$ :

$$
F_{i, j}(u)=\frac{1}{h^{2}}\left(u_{i+1, j}+u_{i, j+1}-4 u_{i, j}+u_{i, j-1}+u_{i-1, j}\right)+\lambda e^{u_{i, j}}=0 .
$$


The Jacobian is now a $(N-2)^{2} \times(N-2)^{2}$ block diagonal matrix of the form:

$$
\nabla F=\frac{1}{h^{2}}\left[\begin{array}{ccccccc}
T_{2} & I & 0 & 0 & \ldots & \ldots & 0 \\
I & T_{3} & I & 0 & \ldots & \ldots & 0 \\
0 & I & T_{4} & I & 0 & \ldots & 0 \\
\vdots & \vdots & \ddots & \ddots & \ddots & \ddots & \vdots \\
0 & \ldots & \ldots & \ldots & \ldots & I & T_{N-1}
\end{array}\right],
$$

where $I$ is the $(N-2) \times(N-2)$ identity matrix and $T_{i}$ is the $(N-2) \times(N-2)$ tridiagonal matrix with non null elements on the $j$-th row:

$$
1, \quad-4+h^{2} \lambda e^{u_{i+j, i+j}}, \quad 1
$$

Regarding the FEM solution, for the one-dimensional Bratu problem, Eq. (16) gives:

$$
R_{k}=\int_{\Omega}\left(\frac{\partial^{2} u}{\partial x^{2}}+\lambda e^{u(x)}\right) \phi_{k}(x) d x .
$$

By inserting Eq. (15) into Eq. (40) and by applying the Green's formula for integration, we get:

$$
R_{k}=\left.\phi_{k}(x) \frac{d u}{d x}\right|_{0} ^{1}-\sum_{j=1}^{N} u_{j} \int_{0}^{1} \frac{d \phi_{j}(x)}{d x} \frac{d \phi_{k}(x)}{d x} d x+\lambda \int_{0}^{1} e^{\sum_{j=1}^{N} u_{j} \phi_{j}(x)} \phi_{k}(x) d x
$$

and because of the zero Dirichlet boundary conditions, Eq. (41) becomes:

$$
R_{k}=-\sum_{j=1}^{N} u_{j} \int_{0}^{1} \frac{d \phi_{j}(x)}{d x} \frac{d \phi_{k}(x)}{d x} d x+\lambda \int_{0}^{1} e^{\sum_{j=1}^{N} u_{j} \phi_{j}(x)} \phi_{k}(x) d x .
$$

The elements of the Jacobian matrix are given by:

$$
\frac{\partial R_{k}}{\partial u_{j}}=-\int_{0}^{1} \frac{d \phi_{j}(x)}{d x} \frac{d \phi_{k}(x)}{d x} d x+\lambda \int_{0}^{1} e^{\sum_{j=1}^{N} u_{j} \phi_{j}(x)} \phi_{j}(x) \phi_{k}(x) d x
$$

Due to the Dirichlet boundary conditions, Eq. (42) becomes:

$$
\left[\begin{array}{cccccc}
1 & 0 & \ldots & 0 & \ldots & 0 \\
\frac{\partial R_{2}}{\partial u_{1}} & \frac{\partial R_{2}}{\partial u_{2}} & \ldots & \frac{R_{2}}{\partial u_{j}} & \ldots & \frac{R_{2}}{\partial u_{N}} \\
\vdots & \vdots & \ddots & \vdots & \ddots & \vdots \\
\frac{\partial R_{k}}{\partial u_{1}} & \frac{\partial R_{k}}{\partial u_{2}} & \ldots & \frac{R_{k}}{\partial u_{j}} & \ldots & \frac{R_{k}}{\partial u_{N}} \\
\vdots & \vdots & \ddots & \vdots & \ddots & \vdots \\
0 & 0 & \ldots & 0 & \ldots & 1
\end{array}\right]_{u^{(n)}}\left[\begin{array}{c}
d u_{1}^{(n)} \\
d u_{2}^{(n)} \\
\vdots \\
d u_{j}^{(n)} \\
\vdots \\
d u_{N}^{(n)}
\end{array}\right]=-\left[\begin{array}{c}
0 \\
R_{2} \\
\vdots \\
R_{k} \\
\vdots \\
0
\end{array}\right]_{\mid u^{(n)}}
$$

For the two-dimensional Bratu problem, the residuals are given by:

$$
R_{k}=\iint_{\Omega}\left(\frac{\partial^{2} u(x, y)}{\partial x^{2}}+\frac{\partial^{2} u(x, y)}{\partial y^{2}}+\lambda e^{u(x, y)}\right) \phi_{k}(x, y) d x d y .
$$


By applying the Green's formula for integration, we get:

$$
\begin{aligned}
R_{k}= & \oint_{\partial \Omega} \nabla u(x, y) d \ell-\iint_{\Omega} \nabla u(x, y) \nabla \phi_{k}(x, y) d x d y \\
& +\iint_{\Omega} \lambda e^{u(x, y)} \phi_{k}(x, y) d x d y .
\end{aligned}
$$

By inserting Eq. (15) and the zero Dirichlet boundary conditions, we get:

$$
\begin{aligned}
R_{k}= & -\sum_{j=1}^{N} u_{j} \iint_{\Omega} \nabla \phi_{j}(x, y) \nabla \phi_{k}(x, y) d x d y \\
& +\iint_{\Omega} \lambda e^{\sum_{j=1}^{N} u_{j} \phi_{j}(x, y)} \phi_{k}(x, y) d x d y .
\end{aligned}
$$

Thus, the elements of the Jacobian matrix for the two-dimensional Bratu problem are given by:

$$
\begin{aligned}
\frac{\partial R_{k}}{\partial u_{j}}= & -\iint_{\Omega} \nabla \phi_{j}(x, y) \nabla \phi_{k}(x, y) d x d y \\
& +\iint_{\Omega} \lambda e^{\sum_{j=1}^{N} u_{j} \phi_{j}(x, y)} \phi_{j}(x, y) \phi_{k}(x, y) d x d y .
\end{aligned}
$$

As before, for our computations we have used quadratic basis functions using an affine element mapping in the domain $[0,1]^{2}$.

\subsubsection{Numerical Solution with Extreme Learning Machine Collocation}

Collocating the ELM network function (1) in the 1D Bratu problem (37) leads to the following system:

$$
F_{i}(\boldsymbol{w}, \lambda)=\sum_{j=1}^{N} w_{j} \frac{d^{2} \psi_{j}\left(x_{i}\right)}{d x^{2}}+\lambda \exp \left(\sum_{j=1}^{N} w_{j} \psi_{j}\left(x_{i}\right)\right)=0, \quad i=2, \ldots, M-1,
$$

with boundary conditions:

$$
F_{1}(\boldsymbol{w}, \lambda)=\sum_{j=1}^{N} w_{j} \psi_{j}(0)=0, \quad F_{M}(\boldsymbol{w}, \lambda)=\sum_{j=1}^{N} w_{j} \psi_{j}(1)=0 .
$$

Thus, the elements of the Jacobian matrix $\nabla_{w} \boldsymbol{F}$ are given by:

$$
\frac{\partial F_{i}}{\partial w_{j}}=\frac{d^{2} \psi_{j}\left(x_{i}\right)}{d x^{2}}+\lambda \psi_{j}\left(x_{i}\right) \exp \left(\sum_{j=1}^{N} w_{j} \psi_{j}\left(x_{i}\right)\right), \quad i=2, \ldots, M-1,
$$

and

$$
\frac{\partial F_{1}}{\partial w_{j}}(\boldsymbol{w}, \lambda)=\psi_{j}(0) \quad \frac{\partial F_{M}}{\partial w_{j}}(\boldsymbol{w}, \lambda)=\psi_{j}(1) .
$$

The application of Newton's method (11) is straightforward using the exact computation of derivatives of the basis functions (see (4) and (7)). 
For the two-dimensional Bratu problem (37), we have:

$$
\begin{aligned}
F_{i}(\boldsymbol{w}, \lambda)= & \sum_{j=1}^{N} w_{j} \frac{\partial^{2} \psi_{j}\left(x_{i}, y_{i}\right)}{\partial x^{2}}+\sum_{j=1}^{N} w_{j} \frac{\partial^{2} \psi_{j}\left(x_{i}, y_{i}\right)}{\partial y^{2}} \\
& +\lambda \exp \left(\sum_{j=1}^{N} w_{j} \psi_{j}\left(x_{i}, y_{i}\right)\right)=0, \quad i=1, \ldots, M_{\Omega}
\end{aligned}
$$

with boundary conditions:

$$
F_{k}(\boldsymbol{w}, \lambda)=\sum_{j=1}^{N} w_{j} \psi_{j}\left(x_{k}, y_{k}\right)=0, \quad k=1, \ldots, M_{1} .
$$

Thus, the elements of the Jacobian matrix $\nabla_{\boldsymbol{w}} F$ read:

$$
\frac{\partial F_{i}}{\partial w_{j}}=\frac{\partial^{2} \psi_{j}\left(x_{i}, y_{i}\right)}{\partial x^{2}}+\frac{\partial^{2} \psi_{j}\left(x_{i}, y_{i}\right)}{\partial y^{2}}+\lambda \psi_{j}\left(x_{i}, y_{i}\right) \exp \left(\sum_{j=1}^{N} w_{j} \psi_{j}\left(x_{i}, y_{i}\right)\right), \quad i=1, \ldots, M_{\Omega}
$$

and

$$
\frac{\partial F_{k}}{\partial w_{j}}(\boldsymbol{w}, \lambda)=\psi_{j}\left(x_{k}, y_{k}\right)=0, \quad k=1, \ldots, M_{1} .
$$

Also in this case, with the above computations the application of Newton's method (11) is straightforward.

\subsubsection{Numerical Results for the One-Dimensional Problem}

First, we show the numerical results for the one-dimensional Liouville-Bratu-Gelfand equation (37) with homogeneous Dirichlet boundary conditions (38). Recall that an exact solution, although in implicit form, is available in this case (see equation (39)); thus, as discussed, the exact solutions are derived using Newton's method with a convergence tolerance of $10^{-12}$. Figure 4 depicts the comparative results between the exact, FD, FEM and ELM solutions on the upper-branch as obtained by applying Newton's iterations, for two values of the parameter $\lambda$ and a fixed $N=40$, namely for $\lambda=3$ close to the turning point (occurring at $\lambda_{c} \sim 3.513830719$ ) and for $\lambda=0.2$. For our illustrations, we have set as initial guess $u_{0}(x)$ a parabola that satisfies the homogeneous boundary conditions, namely:

$$
u_{0}(x)=4 l_{0}\left(x-x^{2}\right),
$$

with a fixed $L_{\infty}$-norm $\|u\|_{\infty}=l_{0}$ close to the one obtained from the exact solution.

In particular, for $\lambda=3$, we used as initial guess a parabola with $l_{0}=2.2$; in all cases Newton's iterations converge to the correct unstable upper-branch solution. For $\lambda=0.2$, we used as initial guess a parabola with $l_{0}=6.4$ (the exact solution has $l_{0} \sim 6.5$ ); again in all cases, Newton's iterations converged to the correct unstable upper-branch solution. To clarify more the behaviour of the convergence, in Fig. 5, we report the regimes of convergence for a grid of $L_{\infty}$ norms of the initial guesses (parabolas) and $\lambda \mathrm{s}$. In Table 4 , we compare the execution times of the four methods when applied for the solution of the Bratu equation (37) with Dirichlet boundary condition (38) and $\lambda=1$. Computations are performed 100 times and we also provide the $5 \%$ and $95 \%$ percentiles. Again, for all practical means, the execution times obtained with the proposed machine learning scheme are comparable with 


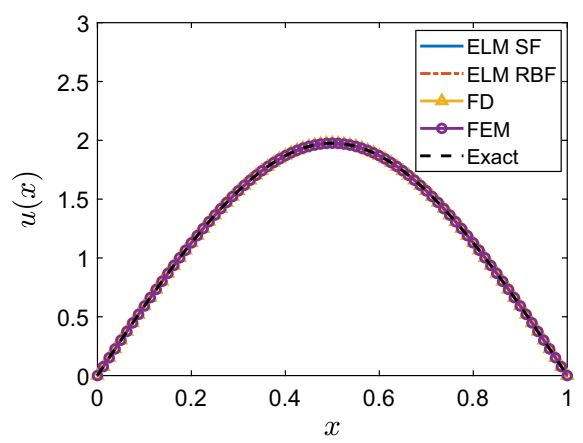

(a)

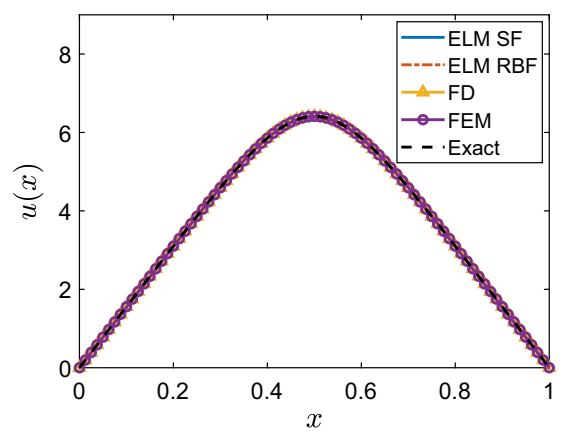

(c)

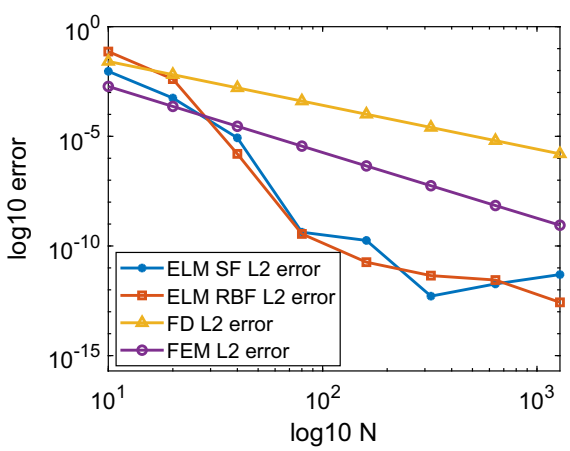

(b)

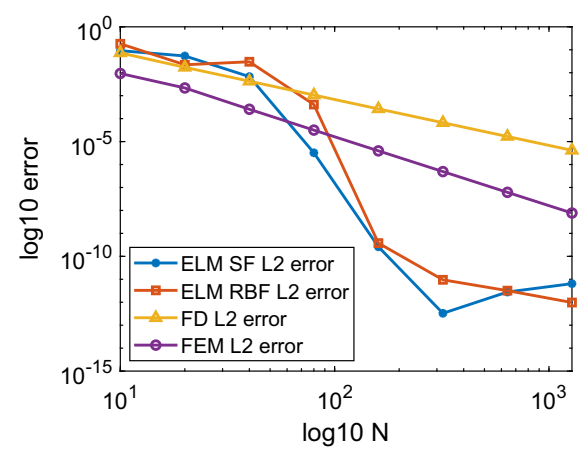

(d)

Fig. 4 Numerical solutions and accuracy of the FD, FEM and the proposed machine learning (ELMs) schemes for the one-dimensional Bratu problem (37). a Computed solutions at the upper-branch unstable solution at $\lambda=3$ for a fixed problem size $N=40$. $\mathbf{b} L_{2}$-norm of differences with respect to the exact unstable solution (39) at $\lambda=3$ for various values of $N$. c Computed solutions at the upper-branch unstable solution at $\lambda=0.2$ with a fixed problem size $N=40$. d $L_{2}$-norm of differences with respect to the exact unstable solution (39) at $\lambda=0.2$ for various values of $N$. The initial guess of the solutions was a parabola satisfying the homogeneous boundary conditions with a fixed $L_{\infty}$-norm $\|u\|_{\infty}=l_{0}$ close to the one resulting from the exact solution

the ones obtained with FD and FEM (note that the execution times obtained with the proposed machine learning scheme are slightly smaller that the ones obtained with FEM), while, as seen, numerical approximation accuracy is better in the ELMs case; the execution times when using the FD are slightly smaller but as shown the FD scheme results to a lower numerical accuracy compared with FEM and the proposed machine learning (ELMs) scheme.

Remark 4 (Linearization of the equation for the numerical solution of the Liouville-BratuGelfand problem)

For the solution of the equation (37) with boundary conditions given by (38), one can consider the following iterative procedure that linearizes the equation:

$$
\left\{\begin{array}{l}
\text { Given } u^{(0)}, \text { do until convergence } \\
\text { find } u^{(k)} \text { such that } \Delta u^{(k)}+\lambda e^{u^{(k-1)}} u^{(k)}=\lambda\left(u^{(k-1)}-1\right) e^{u^{(k-1)}} .
\end{array}\right.
$$

In this way, the nonlinear term becomes a linear reaction term with a non-constant coefficient given by the evaluation of the nonlinearity at the previous step. Then, we implemented fixed point iterations until convergence. Such a linearization procedure is used, for example, in 


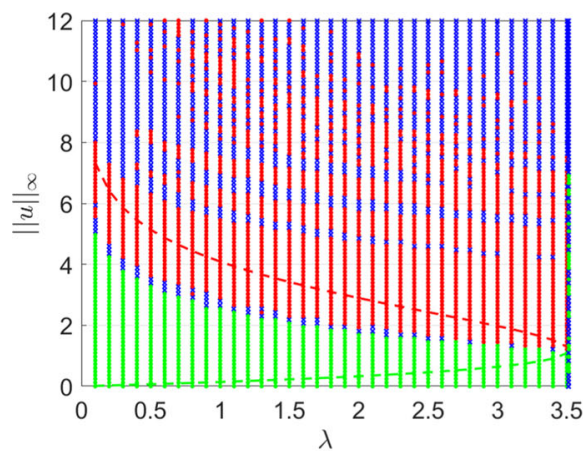

(a)

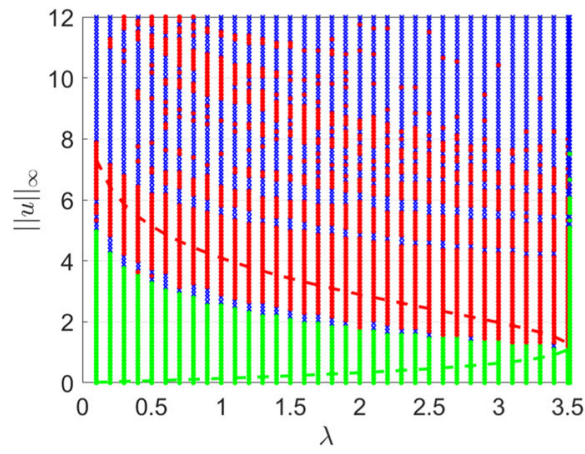

(c)

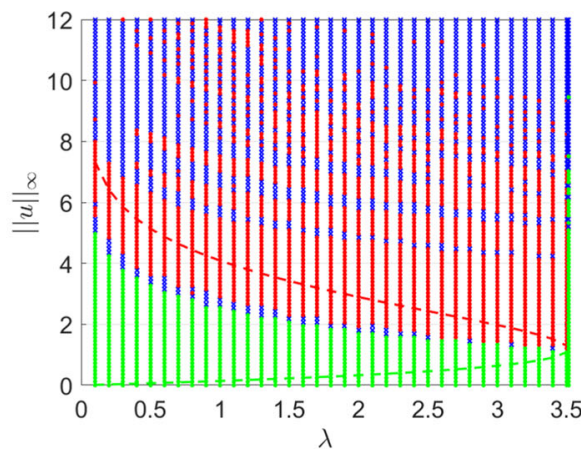

(b)

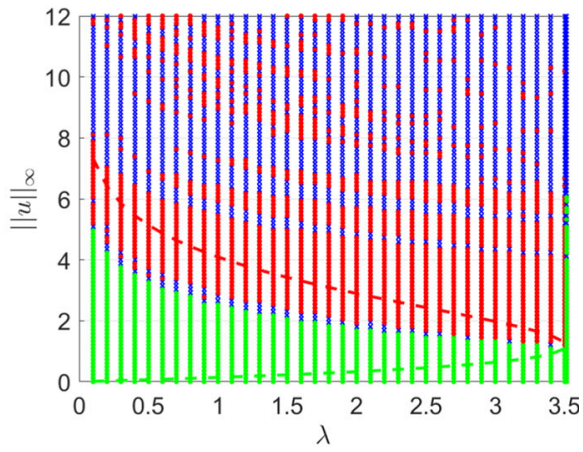

(d)

Fig. 5 Convergence regimes (basin of attraction) of Newton's method with the a FD, b FEM, and c, d ELM numerical schemes for the one-dimensional Bratu problem (37) for a grid of initial guesses $\left(L_{\infty}\right.$-norms of parabolas that satisfy the boundary conditions (38)) and $\lambda$ s. Green points indicate convergence to the lower-branch solutions; Red points indicate convergence to the upper-branch solutions; Blue points indicate divergence. c ELM with logistic SF (3). d ELM with Gaussian RBF (6) (Color figure online)

Table 4 Execution times (s) for the Bratu equation (37) with Dirichlet boundary condition (38) and $\lambda=1$

\begin{tabular}{|c|c|c|c|c|c|c|}
\hline \multirow[t]{2}{*}{$\mathrm{N}$} & \multicolumn{3}{|l|}{ ELM SF } & \multicolumn{3}{|l|}{ ELM RBF } \\
\hline & $5 \%$ & mean & $95 \%$ & $5 \%$ & mean & $95 \%$ \\
\hline 80 & $7.16 \mathrm{e}-03$ & $8.14 \mathrm{e}-03$ & $9.27 \mathrm{e}-03$ & $2.07 \mathrm{e}-03$ & $2.31 \mathrm{e}-03$ & $2.61 \mathrm{e}-03$ \\
\hline 160 & $3.81 \mathrm{e}-02$ & $4.23 \mathrm{e}-02$ & $4.93 e-02$ & $3.53 e-03$ & $4.31 \mathrm{e}-03$ & $4.96 \mathrm{e}-03$ \\
\hline 320 & $1.09 \mathrm{e}-02$ & $1.16 \mathrm{e}-02$ & $1.30 \mathrm{e}-02$ & $7.12 \mathrm{e}-03$ & $7.96 \mathrm{e}-03$ & $8.57 \mathrm{e}-03$ \\
\hline 640 & $3.19 \mathrm{e}-02$ & $3.38 \mathrm{e}-02$ & $3.58 \mathrm{e}-02$ & $2.81 \mathrm{e}-02$ & $3.01 \mathrm{e}-02$ & $3.17 \mathrm{e}-02$ \\
\hline \multirow[t]{2}{*}{$\mathrm{N}$} & \multicolumn{3}{|l|}{ FD } & \multicolumn{3}{|l|}{ FEM } \\
\hline & $5 \%$ & mean & $95 \%$ & $5 \%$ & mean & $95 \%$ \\
\hline 80 & $1.93 e-04$ & $2.60 \mathrm{e}-04$ & $2.65 e-04$ & $2.58 \mathrm{e}-03$ & $2.88 \mathrm{e}-03$ & $3.03 \mathrm{e}-03$ \\
\hline 160 & $5.72 \mathrm{e}-04$ & $7.01 \mathrm{e}-04$ & $8.24 \mathrm{e}-04$ & $5.76 \mathrm{e}-03$ & $6.32 \mathrm{e}-03$ & $6.89 \mathrm{e}-03$ \\
\hline 320 & $1.59 \mathrm{e}-03$ & $1.86 \mathrm{e}-03$ & $2.08 \mathrm{e}-03$ & $1.15 \mathrm{e}-02$ & $1.17 \mathrm{e}-02$ & $1.20 \mathrm{e}-02$ \\
\hline 640 & $8.77 \mathrm{e}-03$ & $9.07 \mathrm{e}-03$ & $9.49 \mathrm{e}-03$ & $3.00 \mathrm{e}-02$ & $3.11 \mathrm{e}-02$ & $3.21 \mathrm{e}-02$ \\
\hline
\end{tabular}




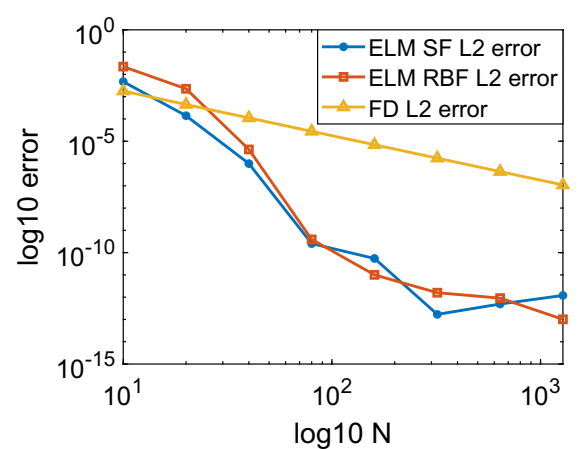

(a)

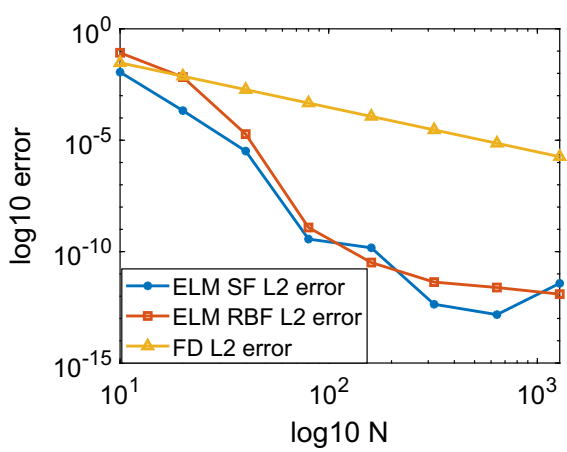

(b)

Fig. 6 Fixed point iterations: $L_{2}$-norm of the difference errors for the low and up branch Liouville-BratuGelfand solution (39) for $\lambda=2$ : a $L_{2}$ errors with respect to $N$ of the low branch solution, b $L_{2}$ errors with respect to $N$ of the upper branch

Table 5 One-dimensional Bratu problem (37)

\begin{tabular}{lllll}
\hline $\mathrm{N}$ & FD & FEM & ELM SF & ELM RBF \\
\hline 20 & $-4.57 \mathrm{e}-03$ & $3.44 \mathrm{e}-05$ & $8.76 \mathrm{e}-05$ & $3.00 \mathrm{eq}-02$ \\
50 & $-7.31 \mathrm{e}-04$ & $8.44 \mathrm{e}-07$ & $2.98 \mathrm{e}-07$ & $6.61 \mathrm{e}-05$ \\
100 & $-1.83 \mathrm{e}-04$ & $5.06 \mathrm{e}-08$ & $-3.71 \mathrm{e}-08$ & $6.13 \mathrm{e}-08$ \\
200 & $-4.57 \mathrm{e}-05$ & $2.36 \mathrm{e}-08$ & $-4.55 \mathrm{e}-09$ & $-2.68 \mathrm{e}-09$ \\
400 & $-1.14 \mathrm{e}-05$ & $1.36 \mathrm{e}-08$ & $2.02 \mathrm{e}-09$ & $2.03 \mathrm{e}-09$ \\
\hline
\end{tabular}

Accuracy of FD, FEM and the proposed machine learning (ELMs) scheme in the approximation of the value of the turning point with respect to the exact value $\lambda=3.513830719125162$. Values express the difference with the computed turning point and the exact one. The value of the turning point was estimated by fitting a parabola around the four points with the largest $\lambda$ values as obtained with arc-length continuation

[36]. In Fig. 6, we report some results on the application of this method. We note that this scheme converges more slowly and it is not so robust compared to the Newton's method.

\subsubsection{Bifurcation Diagram and Numerical Accuracy}

In this section, we report the numerical results obtained by the numerical bifurcation analysis of the one-dimensional Bratu problem (37). Figure 7 shows the constructed bifurcation diagram with respect to the parameter $\lambda$ and in Table 5, we report the accuracy of the computed value as obtained with FD, FEM and the proposed machine learning (ELMs) scheme, versus the exact value of the turning point. As shown, our proposed machine learning scheme provides a bigger numerical accuracy for the value of the turning point for medium to large sizes of the grid, and equivalent results (ELM with SF) to FEM, both outperforming the FD scheme.

In Figs. 8 and 9, we depict the contour plots of the $L_{\infty}$-norms of the differences between the computed solutions by FD, FEM and the proposed machine learning (ELMs) scheme and the exact solutions for the lower-Fig. 8 and upper-branch Fig. 9, respectively with respect to $N$ and $\lambda$. 


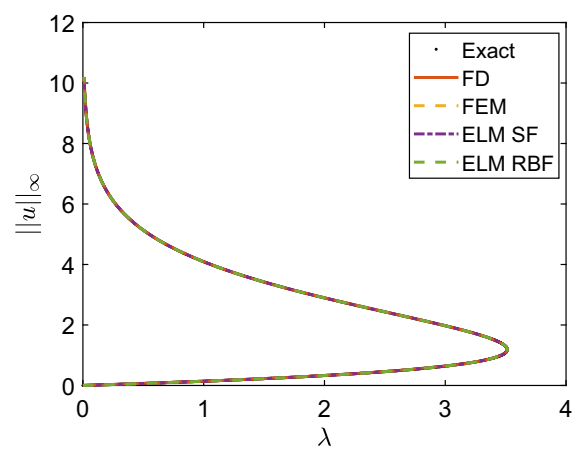

(a)

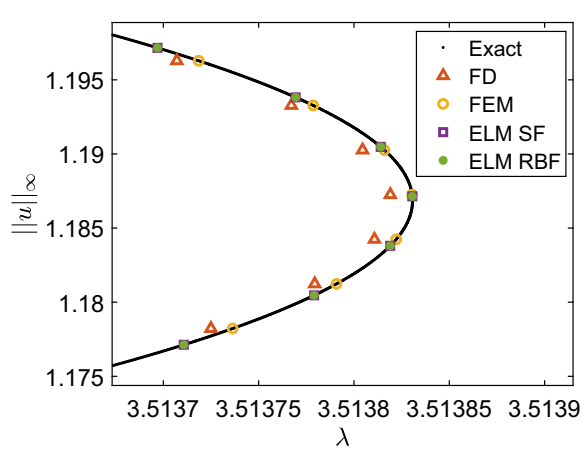

(b)

Fig. 7 a Bifurcation diagram for the one-dimensional Bratu problem (37), with a fixed problem size $N=400$. b Zoom near the turning point

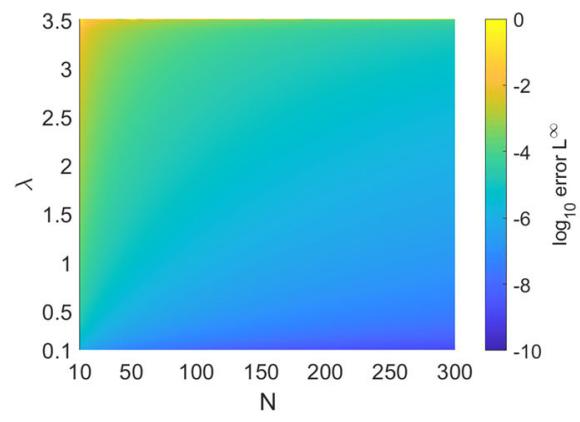

(a)

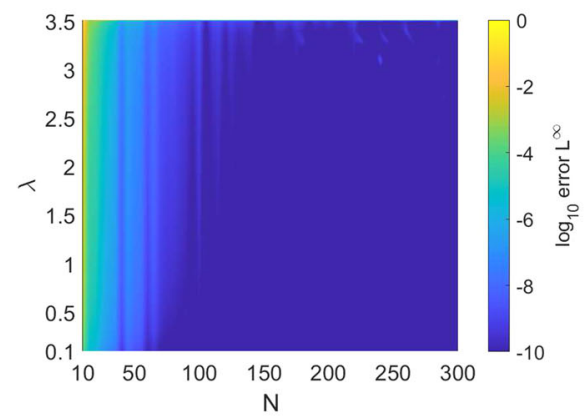

(c)

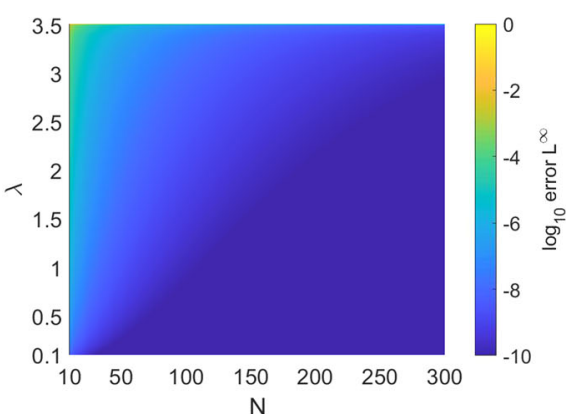

(b)

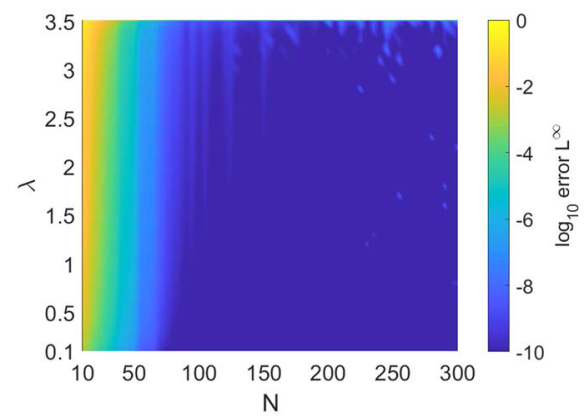

(d)

Fig. 8 One-dimensional Bratu problem (37). Contour plots of the $L_{\infty}$-norms of the differences between the computed and exact (39) solutions for the lower stable branch: a FD, b FEM, c ELM with logistic SF (3), d ELM with Gaussian RBF (6) 


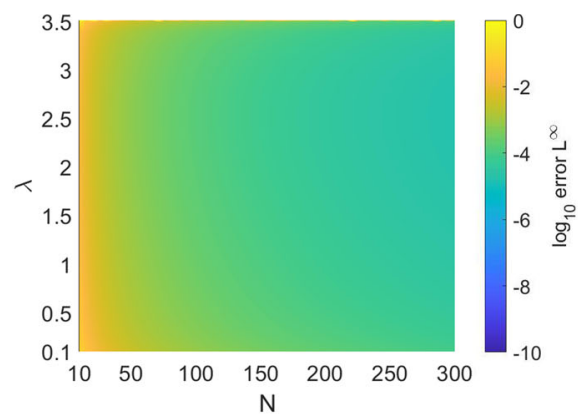

(a)

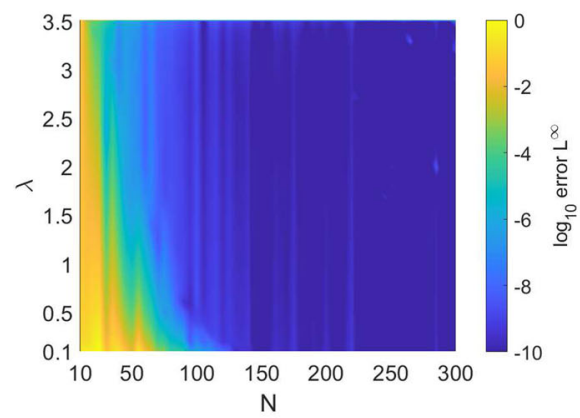

(c)

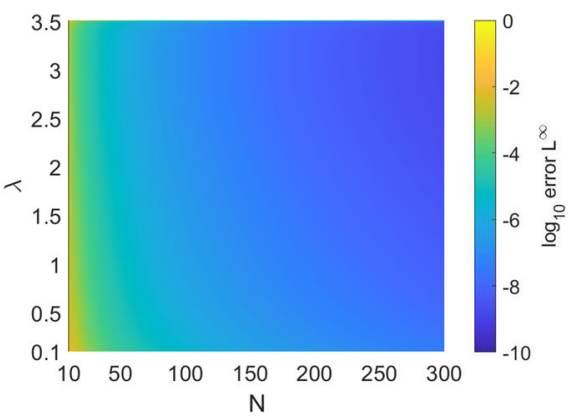

(b)

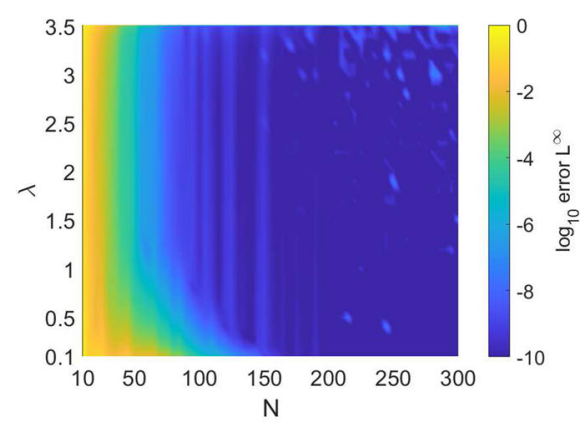

(d)

Fig. 9 One-dimensional Bratu problem (37). Contour plots of the $L_{\infty}$-norms of the differences between the computed and exact (39) solutions for the upper unstable branch: a FD, b FEM, c ELM with logistic SF (3), d ELM with Gaussian RBF (6)

As it is shown, the proposed machine learning schemes outperform both FD and FEM methods for medium to large problem sizes $N$, and provide equivalent results with FEM for low to medium problem sizes, thus both (FEM and the proposed machine learning (ELMs) scheme) outperforming the FD scheme.

\subsubsection{Numerical Results for the Two-Dimensional Problem}

For the two-dimensional problem (37)-(38), no exact analytical solution is available. Thus, for comparing the numerical accuracy of the FD, FEM and the proposed machine learning (ELM) schemes, we considered the value of the bifurcation point that has been reported in key works as discussed in Sect. 4.2. Figure 10 depicts the computed bifurcation diagram as computed via pseudo-arc-length continuation (see Sect. 3). Table 6, summarizes the computed values of the turning point as estimated with the FD, FEM and ELM schemes for various sizes $N$ of the grid.

Remark 5 (The Gelfand-Bratu model) The Liouville-Bratu-Gelfand equation (37) in a unitary ball $B \subset \mathbb{R}^{d}$ with homogeneous Dirichlet boundary conditions is usually refereed as Gelfand-Bratu model. Such equation posses radial solutions $u(r)$ of the one-dimensional 


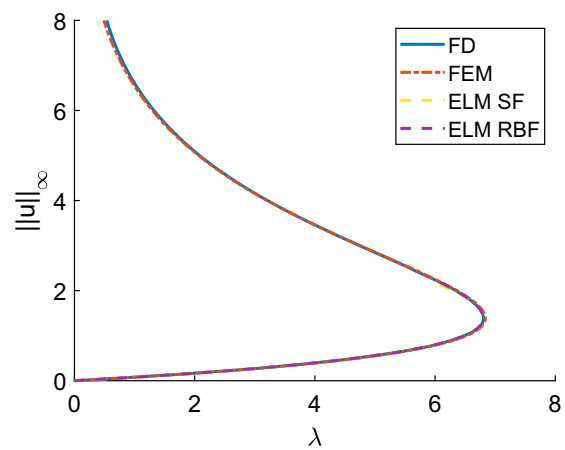

(a)

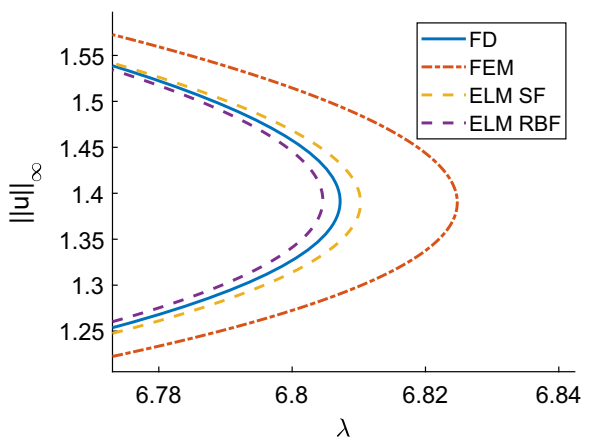

(b)

Fig. 10 a Computed bifurcation diagram for the two-dimensional Bratu problem (37), with a grid of $40 \times 40$ points. b Zoom near the turning point

Table 6 Turning point estimation of the two-dimensional Bratu problem

\begin{tabular}{llllll}
\hline $\mathrm{N}$ & Grid & FD & FEM & ELM SF & ELM RBF \\
\hline 64 & $8 \times 8$ & 6.783434 & 7.083742 & 6.845015 & 7.207203 \\
100 & $10 \times 10$ & 6.792626 & 6.984260 & 6.723902 & 6.930798 \\
196 & $14 \times 14$ & 6.800361 & 6.900313 & 6.855055 & 6.882435 \\
400 & $20 \times 20$ & 6.804392 & 6.856401 & 6.799440 & 6.829754 \\
784 & $28 \times 28$ & 6.806235 & 6.835771 & 6.801689 & 6.806149 \\
1600 & $40 \times 40$ & 6.807220 & 6.824770 & 6.806899 & 6.804600
\end{tabular}

The value that has been reported in the literature in key works (see e.g. [9]) is $\lambda^{*}=6.808124$. The value of the turning point was estimated by fitting a parabola around the four points with the largest $\lambda$ values as obtained by the arc-length continuation

non-linear boundary-value problem [58]:

$$
\left\{\begin{array}{l}
u^{\prime \prime}(r)+\frac{d-1}{r} u^{\prime}(r)+\lambda e^{u(r)}=0 \quad 0<r<1 \\
u(1)=u^{\prime}(0)=0
\end{array}\right.
$$

In the case $d=2$ this equation gives multiple solutions if $\lambda<\lambda_{c}=2$. For example, in [53], the authors have used Mathematica to give analytical solutions at various values of $\lambda$; for our tests we consider:

$$
\begin{aligned}
& \lambda=\frac{1}{2} \rightarrow u(r)=\log \left(\frac{16(7+4 \sqrt{3})}{\left(7+4 \sqrt{3}+r^{2}\right)^{2}}\right) \\
& \lambda=1 \rightarrow u(r)=\log \left(\frac{8(3+2 \sqrt{2})}{\left(3+2 \sqrt{2}+r^{2}\right)^{2}}\right) .
\end{aligned}
$$

Figure 11 depicts the numerical accuracy of the proposed machine learning (ELM) collocation scheme with respect to the exact solutions for two values of $\lambda$, namely for $\lambda=1 / 2$ and for $\lambda=1$. Because no meshing procedure is involved, and because the collocation equation 


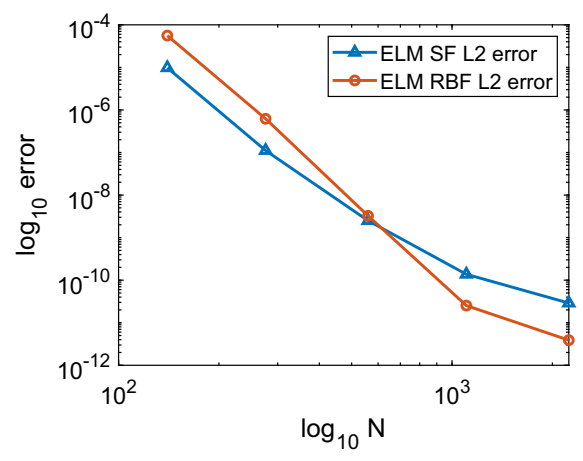

(a)

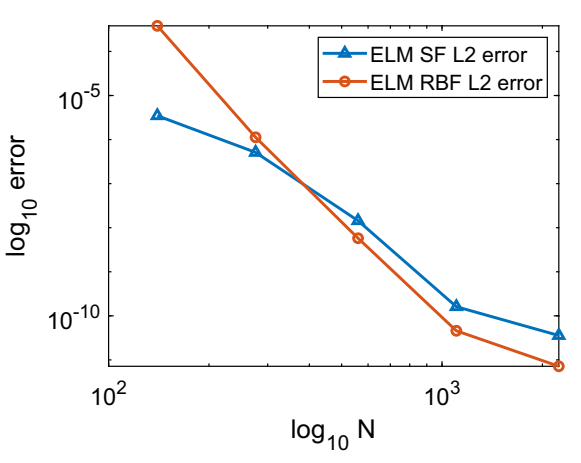

(b)

Fig. 11 Numerical accuracy of ELMs for the radial two-dimensional Gelfand-Bratu problem (44). $L_{2}$-norm of differences of the analytical solutions (45) w.r.t. the number of neurons $N$ in ELMs with both logistic SF (3) and Gaussian RBF (6): $\mathbf{a} \lambda=1 / 2, \mathbf{b} \lambda=1$

seeks no other point, the implementation of the Newton's method is straightforward when changing the geometry of the domain.

\section{Conclusions}

We proposed a machine learning numerical method based on Extreme Learning Machines (ELMs) and collocation for the approximation of steady-state solutions of non-linear PDEs. The proposed numerical scheme takes advantage of the property of the ELMs as universal function approximators, bypassing the need of the computational very expensive - and most-of-the times without any guarantee for convergence of-the training phase of other types of machine learning such as single or multilayer ANNs and Deep-learning networks. The base of the approximation subspace on which a solution of the PDE is sought are the randomized transfer functions of the hidden layer which are weighted by the only unknown parameters, that is the weights of the hidden to output layer. For linear PDEs, these can be computed by solving a linear regularization problem in one step. In our previous work [8], we demonstrated that ELMs can provide robust and accurate approximations of the steady-state solution of benchmark linear PDEs with steep gradients, for which analytical solutions were available. Here, we address a new numerical scheme based on ELMs that can be used to solve steady state problems of non-linear PDEs, and by bridging them with numerical continuation methods, we show how one can exploit the arsenal of numerical bifurcation theory to trace branches of solutions past turning points. For our demonstrations, we considered two celebrated classes of nonlinear PDEs whose solutions bifurcate as parameter values change: the one-dimensional viscous Burgers equation (a fundamental representative of advectiondiffusion PDEs) and the one- and two-dimensional Liouville-Bratu-Gelfand equation (a fundamental representative of reaction-diffusion PDEs). By coupling the proposed numerical scheme with Newton-Raphson iterations and the "pseudo" arc-length continuation method, we constructed the corresponding bifurcation diagrams past turning points. The efficiency of the proposed numerical machine learning collocation method was compared against two of the most established numerical solution methods, namely central Finite Differences and Galerkin Finite Elements. By doing so, we showed that (for the same problem size) the pro- 
posed machine-learning approach outperforms FD and FEM schemes for relatively medium to large sizes of the grid, both with respect to the accuracy of the computed solutions for a wide range of the bifurcation parameter values and the approximation accuracy of the turning points. Thus, we show that the computational times of the proposed machine learning method are comparable with the ones obtained with the other two schemes (actually the computational times obtained with the proposed method are slightly smaller than the ones obtained with FEM; the FD scheme provides slightly smaller times but fails to approximate solutions with steep gradients and in general results to poorer numerical approximations). Hence, the proposed method arises as an alternative and powerful new numerical technique for the approximation of steady-state solutions of non-linear PDEs. Furthermore, its implementation is far simpler than the implementation of FEM, thus providing equivalent or even better numerical accuracy, and in all cases is shown to outperform the simple FD scheme, which fails to approximate steep gradients as here arise near the boundaries. Of course there are many open problems linked to the implementation of the proposed numerical method that ask for further and deeper investigation, such as the theoretical investigation of the impact of the type of transfer functions and the probability distribution of their parameter values functions to the approximation of the solutions. Further directions could be towards the extension of the method for the solution of time-dependent non-linear PDEs as well as the solution of inverse-problems in PDEs. Finally, we should note that the aim of the current work was to show how random projection neural networks and namely, ELMs can be efficiently used for the numerical solution of steady-state problems of nonlinear PDEs, the comparison of the performance of the proposed method with other traditional methods such as FD and FEM and by exploiting the tools of numerical bifurcation theory, the construction of the corresponding bifurcation diagrams. Thus, a comparison with other machine learning approaches such as deep-learning or other schemes that have been used for the numerical solution of time-dependent PDEs (see e.g. $[18,52]$ ) is out of the scope of this work. Such a comparison is still a challenging task that we aim to confront in a future work.

Acknowledgements Gianluca Fabiani is supported by a 4-year scholarship by the Scuola Superiore Meridionale, Ph.D. Program in Modeling and Engineering Risk and Complexity, Università degli Studi di Napoli Federico II, Italy Francesco Calabrò and Constantinos Siettos were partially supported by INdAM, through GNCS research projects. Constantinos Siettos acknowledges also support by the Italian program Fondo Integrativo Speciale per la Ricerca (FISR) - B55F20002320001.

Funding Open access funding provided by Università degli Studi di Napoli Federico II within the CRUI-CARE Agreement.

Data availability Not applicable.

\section{Declarations}

Conflict of interest Not applicable.

Code availability The code will be made available upon publication of the manuscript.

Open Access This article is licensed under a Creative Commons Attribution 4.0 International License, which permits use, sharing, adaptation, distribution and reproduction in any medium or format, as long as you give appropriate credit to the original author(s) and the source, provide a link to the Creative Commons licence, and indicate if changes were made. The images or other third party material in this article are included in the article's Creative Commons licence, unless indicated otherwise in a credit line to the material. If material is not included in the article's Creative Commons licence and your intended use is not permitted by statutory 
regulation or exceeds the permitted use, you will need to obtain permission directly from the copyright holder. To view a copy of this licence, visit http://creativecommons.org/licenses/by/4.0/.

\section{References}

1. Allen, E.J., Burns, J.A., Gilliam, D.S.: Numerical approximations of the dynamical system generated by burgers' equation with neumann-dirichlet boundary conditions. ESAIM: Mathematical Modelling and Numerical Analysis-Modélisation Mathématique et Analyse Numérique 47(5), 1465-1492 (2013)

2. Asprone, D., Auricchio, F., Manfredi, G., Prota, A., Reali, A., Sangalli, G.: Particle methods for a $1 \mathrm{~d}$ elastic model problem: Error analysis and development of a second-order accurate formulation. Computer Modeling in Engineering \& Sciences (CMES) 62(1), 1-21 (2010)

3. Auricchio, F., Da Veiga, L.B., Hughes, T.J., Reali, A., Sangalli, G.: Isogeometric collocation for elastostatics and explicit dynamics. Computer methods in applied mechanics and engineering 249, 2-14 (2012)

4. Bai, Z., Huang, G.B., Wang, D., Wang, H., Westover, M.B.: Sparse extreme learning machine for classification. IEEE transactions on cybernetics 44(10), 1858-1870 (2014)

5. Benton, E.R., Platzman, G.W.: A table of solutions of the one-dimensional burgers equation. Quarterly of Applied Mathematics 30(2), 195-212 (1972)

6. Boyd, J.P.: An analytical and numerical study of the two-dimensional bratu equation. Journal of Scientific Computing 1(2), 183-206 (1986)

7. Brezzi, F., Rappaz, J., Raviart, P.A.: Finite dimensional approximation of nonlinear problems. Numerische Mathematik 38(1), 1-30 (1982)

8. Calabrò, F., Fabiani, G., Siettos, C.: Extreme learning machine collocation for the numerical solution of elliptic pdes with sharp gradients. arXiv preprint arXiv:2012.05871 (2020)

9. Chan, T.F., Keller, H.: Arc-length continuation and multigrid techniques for nonlinear elliptic eigenvalue problems. SIAM Journal on Scientific and Statistical Computing 3(2), 173-194 (1982)

10. Chan-Wai-Nam, Q., Mikael, J., Warin, X.: Machine learning for semi linear pdes. Journal of Scientific Computing 79(3), 1667-1712 (2019)

11. Chaturvedi, I., Ragusa, E., Gastaldo, P., Zunino, R., Cambria, E.: Bayesian network based extreme learning machine for subjectivity detection. Journal of The Franklin Institute 355(4), 1780-1797 (2018)

12. Chen, J., Zeng, Y., Li, Y., Huang, G.B.: Unsupervised feature selection based extreme learning machine for clustering. Neurocomputing 386, 198-207 (2020)

13. Cliffe, K., Spence, A., Tavener, S.: The numerical analysis of bifurcation problems with application to fluid mechanics. Acta Numerica 9(00), 39-131 (2000)

14. Dai, H., Cao, J., Wang, T., Deng, M., Yang, Z.: Multilayer one-class extreme learning machine. Neural Networks 115, 11-22 (2019)

15. Dhooge, A., Govaerts, W., Kuznetsov, Y.A., Meijer, H.G.E., Sautois, B.: New features of the software matcont for bifurcation analysis of dynamical systems. Mathematical and Computer Modelling of Dynamical Systems 14(2), 147-175 (2008)

16. Doedel, E., Tuckerman, L.S.: Numerical methods for bifurcation problems and large-scale dynamical systems, vol. 119. Springer Science \& Business Media (2012)

17. Doedel, E.J., Champneys, A.R., Dercole, F., Fairgrieve, T.F., Kuznetsov, Y.A., Oldeman, B., Paffenroth, R., Sandstede, B., Wang, X., Zhang, C.: Auto-07p: Continuation and bifurcation software for ordinary differential equations. Available for download from http://indy.cs.concordia.ca/auto (2007)

18. Dong, S., Li, Z.: Local extreme learning machines and domain decomposition for solving linear and nonlinear partial differential equations. arXiv preprint arXiv:2012.02895 (2020)

19. Dong, S., Li, Z.: A modified batch intrinsic plasticity method for pre-training the random coefficients of extreme learning machines. arXiv preprint arXiv:2103.08042 (2021)

20. Dwivedi, V., Srinivasan, B.: Physics informed extreme learning machine (pielm)-a rapid method for the numerical solution of partial differential equations. Neurocomputing 391, 96-118 (2020)

21. Fresca, S., Dede, L., Manzoni, A.: A comprehensive deep learning-based approach to reduced order modeling of nonlinear time-dependent parametrized pdes. Journal of Scientific Computing 87(61),(2021)

22. Gebhardt, C.G., Steinbach, M.C., Schillinger, D., Rolfes, R.: A framework for data-driven structural analysis in general elasticity based on nonlinear optimization: The dynamic case. International Journal for Numerical Methods in Engineering 121(24), 5447-5468 (2020)

23. Glowinski, R., Keller, H.B., Reinhart, L.: Continuation-conjugate gradient methods for the least squares solution of nonlinear boundary value problems. SIAM journal on scientific and statistical computing 6(4), 793-832 (1985) 
24. González-García, R., Rico-Martìnez, R., Kevrekidis, I.G.: Identification of distributed parameter systems: A neural net based approach. Computers \& chemical engineering 22, S965-S968 (1998)

25. Govaerts, W.J.: Numerical methods for bifurcations of dynamical equilibria. SIAM (2000)

26. Hadash, G., Kermany, E., Carmeli, B., Lavi, O., Kour, G., Jacovi, A.: Estimate and replace: A novel approach to integrating deep neural networks with existing applications. arXiv preprint arXiv:1804.09028 (2018)

27. Hajipour, M., Jajarmi, A., Baleanu, D.: On the accurate discretization of a highly nonlinear boundary value problem. Numerical Algorithms 79(3), 679-695 (2018)

28. Han, J., Jentzen, A., Weinan, E.: Solving high-dimensional partial differential equations using deep learning. Proceedings of the National Academy of Sciences 115(34), 8505-8510 (2018)

29. Huang, G., Huang, G.B., Song, S., You, K.: Trends in extreme learning machines: A review. Neural Networks 61, 32-48 (2015)

30. Huang, G., Kasun, L., Zhou, H., Vong, C.: Representational learning with extreme learning machine for big data. IEEE Intelligent Systems 28(6), 31-34 (2013)

31. Huang, G., Zhou, H., Ding, X., Zhang, R.: Extreme learning machine for regression and multiclass classification. IEEE Transactions on Systems, Man, and Cybernetics, Part B (Cybernetics) 42(2), 513529 (2012). https://doi.org/10.1109/TSMCB.2011.2168604

32. Huang, G.B., Ding, X., Zhou, H.: Optimization method based extreme learning machine for classification. Neurocomputing 74(1-3), 155-163 (2010)

33. Huang, G.B., Zhou, H., Ding, X., Zhang, R.: Extreme learning machine for regression and multiclass classification. IEEE Transactions on Systems, Man, and Cybernetics, Part B (Cybernetics) 42(2), 513-529 (2011)

34. Huang, G.B., Zhu, Q.Y., Siew, C.K.: Extreme learning machine: theory and applications. Neurocomputing 70(1-3), 489-501 (2006)

35. Husmeier, D.: Random vector functional link (rvfl) networks. In: Neural Networks for Conditional Probability Estimation, pp. 87-97. Springer (1999)

36. Iqbal, S., Zegeling, P.A.: A numerical study of the higher-dimensional gelfand-bratu model. Computers \& Mathematics with Applications 79(6), 1619-1633 (2020)

37. Jaeger, H.: Adaptive nonlinear system identification with echo state networks. Advances in neural information processing systems 15, 609-616 (2002)

38. Jaeger, H., Haas, H.: Harnessing nonlinearity: Predicting chaotic systems and saving energy in wireless communication. science 304(5667), 78-80 (2004)

39. Kelley, C.T.: Numerical methods for nonlinear equations. Acta Numerica 27, 207-287 (2018). https:// doi.org/10.1017/S0962492917000113

40. Krauskopf, B., Osinga, H.M., Galán-Vioque, J.: Numerical continuation methods for dynamical systems, vol. 2. Springer (2007)

41. Kuznetsov, Y.A.: Elements of applied bifurcation theory, vol. 112. Springer Science \& Business Media (2013)

42. Lagaris, I.E., Likas, A., Fotiadis, D.I.: Artificial neural networks for solving ordinary and partial differential equations. IEEE transactions on neural networks 9(5), 987-1000 (1998)

43. Mohsen, A.: A simple solution of the bratu problem. Computers \& Mathematics with Applications 67(1), 26-33 (2014)

44. Olson, L.G., Georgiou, G.C., Schultz, W.W.: An efficient finite element method for treating singularities in laplace's equation. Journal of Computational Physics 96(2), 391-410 (1991)

45. Ozturk, M.C., Xu, D., Principe, J.C.: Analysis and design of echo state networks. Neural computation 19(1), 111-138 (2007)

46. Panghal, S., Kumar, M.: Optimization free neural network approach for solving ordinary and partial differential equations. Engineering with Computers pp. 1-14 (2020)

47. Pao, Y.H., Park, G.H., Sobajic, D.J.: Learning and generalization characteristics of the random vector functional-link net. Neurocomputing 6(2), 163-180 (1994)

48. Paquot, Y., Duport, F., Smerieri, A., Dambre, J., Schrauwen, B., Haelterman, M., Massar, S.: Optoelectronic reservoir computing. Scientific reports 2(1), 1-6 (2012)

49. Pinkus, A.: Approximation theory of the mlp model. Acta Numerica 1999: Volume 8 8, 143-195 (1999)

50. Quarteroni, A., Valli, A.: Numerical approximation of partial differential equations, vol. 23. Springer Science \& Business Media (2008)

51. Raissi, M., Perdikaris, P., Karniadakis, G.E.: Numerical gaussian processes for time-dependent and nonlinear partial differential equations. SIAM Journal on Scientific Computing 40(1), A172-A198 (2018)

52. Raissi, M., Perdikaris, P., Karniadakis, G.E.: Physics-informed neural networks: A deep learning framework for solving forward and inverse problems involving nonlinear partial differential equations. Journal of Computational Physics 378, 686-707 (2019) 
53. Raja, M.A.Z., Samar, R., et al.: Neural network optimized with evolutionary computing technique for solving the 2-dimensional bratu problem. Neural Computing and Applications 23(7), 2199-2210 (2013)

54. Sakemi, Y., Morino, K., Leleu, T., Aihara, K.: Model-size reduction for reservoir computing by concatenating internal states through time. Scientific reports 10(1), 1-13 (2020)

55. Samaniego, E., Anitescu, C., Goswami, S., Nguyen-Thanh, V.M., Guo, H., Hamdia, K., Zhuang, X., Rabczuk, T.: An energy approach to the solution of partial differential equations in computational mechanics via machine learning: Concepts, implementation and applications. Computer Methods in Applied Mechanics and Engineering 362, 112790 (2020)

56. Schilder, F., Dankowicz, H.: Continuation core and toolboxes (coco). Source-Forge. net, project cocotools (2017)

57. Schmidt, W.F., Kraaijveld, M.A., Duin, R.P., et al.: Feed forward neural networks with random weights. In: International Conference on Pattern Recognition, pp. 1-1. IEEE COMPUTER SOCIETY PRESS (1992)

58. Syam, M.I.: The modified broyden-variational method for solving nonlinear elliptic differential equations. Chaos, Solitons \& Fractals 32(2), 392-404 (2007)

59. Tang, J., Deng, C., Huang, G.B.: Extreme learning machine for multilayer perceptron. IEEE transactions on neural networks and learning systems 27(4), 809-821 (2015)

60. Tissera, M.D., McDonnell, M.D.: Deep extreme learning machines: supervised autoencoding architecture for classification. Neurocomputing 174, 42-49 (2016)

61. Wang, Y., Cao, F., Yuan, Y.: A study on effectiveness of extreme learning machine. Neurocomputing 74(16), 2483-2490 (2011)

62. Wei, Q., Jiang, Y., Chen, J.Z.: Machine-learning solver for modified diffusion equations. Physical Review E 98(5), 053304 (2018)

Publisher's Note Springer Nature remains neutral with regard to jurisdictional claims in published maps and institutional affiliations. 